Portland State University

PDXScholar

Fall 12-10-2018

\title{
Accumulation and Transmission Dynamics of a Naturally-Occurring mtDNA Deletion in Caenorhabditis briggsae
}

Jennifer Anne Sullins

Portland State University

Follow this and additional works at: https://pdxscholar.library.pdx.edu/open_access_etds

Part of the Biology Commons, Evolution Commons, and the Genetics Commons Let us know how access to this document benefits you.

Recommended Citation

Sullins, Jennifer Anne, "Accumulation and Transmission Dynamics of a Naturally-Occurring mtDNA Deletion in Caenorhabditis briggsae" (2018). Dissertations and Theses. Paper 4729.

https://doi.org/10.15760/etd.6613

This Thesis is brought to you for free and open access. It has been accepted for inclusion in Dissertations and Theses by an authorized administrator of PDXScholar. Please contact us if we can make this document more accessible: pdxscholar@pdx.edu. 


\title{
Accumulation and Transmission Dynamics of a Naturally-Occurring mtDNA Deletion in Caenorhabditis briggsae
}

\author{
by \\ Jennifer Anne Sullins
}

A thesis submitted in partial fulfillment of the requirements for the degree of

\author{
Master of Science \\ in \\ Biology
}

Thesis Committee:

Suzanne Estes, Chair

Susan Masta

Jason Podrabsky

Portland State University

2018 
(C) 2018 Jennifer Anne Sullins 
Maintaining mitochondrial genome sequence integrity is essential for preserving normal mitochondrial function. Several human diseases have been associated with heteroplasmic mitochondrial genome mutations, but few genetic systems can simultaneously represent pathogenic mitochondrial genome evolution and inheritance. The nematode Caenorhabditis briggsae is one such model. Natural $C$. briggsae isolates are globally-distributed and phylogenetically grouped into three distinct clades, with isolates exhibiting varying levels of a large-scale mtDNA deletion, nad5s. Furthermore, a small subset of clade II isolates exhibits putative compensatory mutations that may reduce the risk of deletion formation and accumulation in those populations. In this thesis, I characterize the dynamics of nad5 $\Delta$ heteroplasmy levels during both development and transmission in several $C$. briggsae natural isolates, including two containing putatively protective compensatory mutations $(\mathrm{C}+)$.

For all isolates tested, nad5 5 heteroplasmy levels increased across nematode development, with L1 (first larval stage) exhibiting the lowest deletion load for all but one isolate that exhibited highly variable nad5 $\Delta$ levels, while the increase was slowest and overall nad5 $\Delta$ levels remained relatively low in $\mathrm{C}+$ isolates. These results support previous work suggesting that $n a d 5 \Delta$ is a selfish element and demonstrate the protective nature of compensatory mutations in inhibiting mtDNA deletion accumulation. 
In nad5 $\Delta$ inheritance assays, $\mathrm{C}+$ isolates displayed a strong pattern of reversion to wildtype mtDNA levels that was not seen in isolates lacking

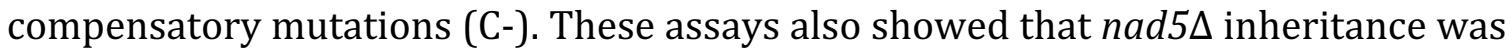
not well predicted by total maternal nad5 5 proportion in either C+ or C- isolates; offspring nad5 $\Delta$ levels were generally much lower than maternal levels, consistent with some form of negative selection operating between generations. Assays of both maternal somatic and gonadal tissues had slightly more power to predict offspring deletion levels than did assays of whole-worm maternal samples; this result likely points to variance in deletion levels originating from an untested parental tissue type present within the whole-worm samples.

This thesis provided deeper insights into the patterns of mtDNA deletion transmission and age-associated dynamics. It was the first project of its type to survey mutation dynamics and heteroplasmy levels of a naturally-occurring large-scale mtDNA deletion. Thus, my work serves to further develop C. briggsae for use as an experimental model of human mtDNA deletion dynamics and mitochondrial dysfunction. 
Many, many thanks to Dr. Suzanne Estes for all her support and assistance during my graduate degree and over the years since I began in her lab, but primarily for her unending belief in me while I am still learning to believe in myself. Thanks to Drs. Susan Masta and Jason Podrabsky for serving on my committee and providing feedback along the way.

Thanks to Anna Coleman-Hulbert and Stephen Christy for being awesome friends and wonderful lab colleagues, providing professional and academic support as well as extracurricular fun; Dr. Dee Denver and Dana Howe at Oregon State University for welcoming me into their lab one summer to teach me PCR techniques; Dr. Lindsay Holden for assisting me in developing a qPCR protocol and answering my multitudinous questions (additional shout out to Dr. Kim Brown's lab for generously allowing use of their qPCR machine); and Alexandra Gallegos for performing a significant number of the qPCR assays.

Thanks to my friends and family for all of their support and encouragement. A very special thank you to my dear friend, Kat Biskind, who is always there for me. Without her insightful guidance and support in all aspects of life, I would not be at this point right now. 
TABLE OF CONTENTS

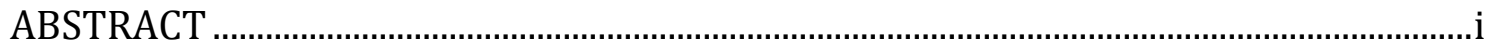

ACKNOWLEDGEMENTS .................................................................................................ii

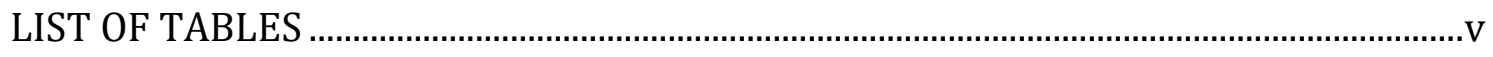

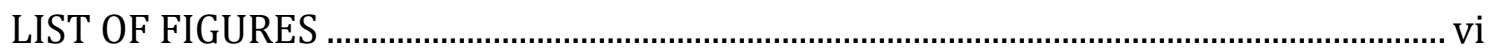

LIST OF ABBREVIATIONS.......................................................................................................vii

CHAPTER 1: Introduction............................................................................................... 1

CHAPTER 2: Accumulation and Transmission Dynamics of a Naturally-Occurring mtDNA Deletion in Caenorhabditis briggsae ................................................................... 4

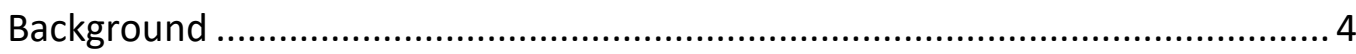

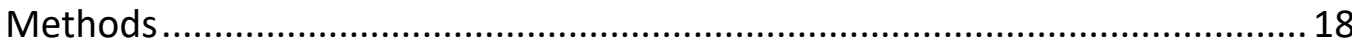

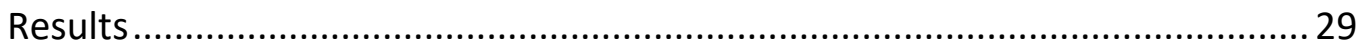

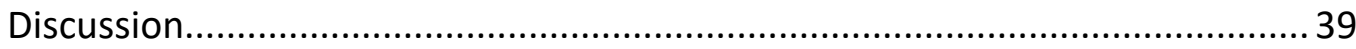

CHAPTER 3: Conclusion .....................................................................................................4

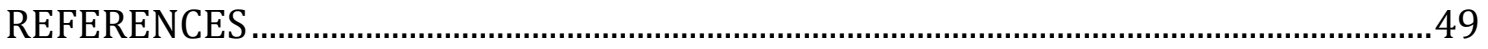




\section{I S T O F T A B L E S}

Table 1. Caenorhabditis briggsae natural isolates used for this study.................... 19

Table 2. PCR banding by isolate by life stage .............................................................

Table 3. PCR banding by isolate by inheritance.......................................................32

Table 4. qPCR mean proportion nad5 5 by isolate by inheritance..............................34 


\section{LIS T OF FIGURES}

Figure 1. Schematic of Caenorhabditis mitochondrial electron transport chain (ETD)

Figure 2. Mitochondrial genome map of congener species Caenorhabditis elegans.. 9

Figure 3. Evolutionary origins of $\psi$ nad5 elements

Figure 4. Unad5 element positions in C. briggsae mitochondrial genomes.

Figure 5. Phylogenetic relationships and nad5 $\Delta$ heteroplasmy levels of $C$. briggsae isolates

Figure 6. Sequence divergence in flanking regions may confer compensatory protection

Figure 7. Phylogenetic relationships of $C$. briggsae natural isolates base on mtDNA, nad5 $\Delta$ locus schematic, and conventional PCR banding possibilities for this region 20

Figure 8. Sampling methods for individual worm collection prior to PCR and qPCR assays

Figure 9. Representative images of gel banding patterns from whole-worm PCR reactions

Figure 10. Representative example of amplification plots from one qPCR assay ....26

Figure 11. Banding score proportions by life stage by isolate and effect likelihood ratio tests

Figure 12. Parent and offspring banding correlation scatterplot by strain 32

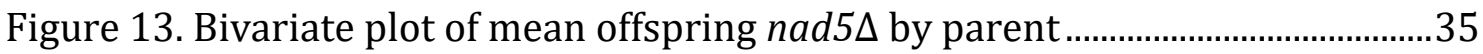

Figure 14. Bivariate plot of mean offspring nad5 $\Delta$ by parent gonad.............................36

Figure 15. Bivariate plot of mean offspring nad5 $\Delta$ by parent head ..............................37

Figure 16. Bivariate plot of parent gonad nad5 $\Delta$ by parent head 38 


\title{
L IS T O F A B B R E V I A T I O N S
}

\author{
ATP - Adenosine triphosphate \\ C+- Compensatory mutation present \\ C- - Compensatory mutation absent \\ Cybrid - Cytoplasmic-nuclear hybrid \\ ETC - Electron transport chain \\ $\Delta \Psi \mathrm{M}$ - Membrane potential \\ mtDNA - Mitochondrial DNA \\ nDNA - Nuclear DNA \\ OXPHOS - Oxidative phosphorylation \\ ROS - Reactive oxygen species \\ rRNA - ribosomal RNA \\ tRNA - transfer RNA
}




\section{H A P T E 1: Introdution}

With efficient natural selection, deleterious mutations are expected to be eliminated from populations. However, "selfish genetic elements" such as segregation distorters exhibit transmission advantages and long-term persistence or accumulation in populations despite their neutral or negative effects on organismal fitness. Previous research from our group has shown that mitochondrial DNA (mtDNA) bearing large function-disrupting deletions demonstrate such selfish evolutionary dynamics under certain conditions (Clark et al. 2012; Ma and O’Farrell 2016).

The mechanism(s) by which mtDNA deletions arise is still debated, but they are generally found in a heteroplasmic state-both mutant and wildtype mtDNAs present within an individual—with biochemical defects manifesting beyond a certain heteroplasmy threshold. Single large-scale mtDNA deletions account for a quarter of all mitochondrial disorders present in adults (Chinnery et al. 2000). Despite wideranging implications of mtDNA selfish elements for human disease and topics in evolutionary biology (e.g., speciation), the forces controlling their formation, agerelated accumulation, and risk of transmission remain largely unknown.

Caenorhabditis briggsae nematodes offer a perfect system for experimental study of mtDNA deletion dynamics (Howe and Denver 2008; Estes et al. 2011; Clark et al. 2012; Hicks et al. 2012; Phillips et al. 2015). Natural C. briggsae populations harbor varying levels of a deletion that eliminates 786 base-pairs of the NADHdehydrogenase-5 (nad5) gene and dozens of highly conserved codons. Wildtype NAD5 is an integral component of complex I of the mitochondrial electron transport 
chain. The deletion, nad5 $\Delta$, is found only in a heteroplasmic state and is thought to result from mispairing between flanking repeats during DNA replication. Importantly, certain C. briggsae isolates contain putative compensatory mutations $(\mathrm{C}+$ isolates $)$ that reduce the similarity of these flanking regions and are hypothesized to reduce $n a d 5 \Delta$-formation events compared to isolates lacking these mutations (Cisolates). However, we still do not know how nad5 $\Delta$ frequency shifts during motheroffspring transmission or with nematode age, and if the presence of compensatory mutations influences these patterns.

My thesis addressed the following two major aims to provide insight into the patterns of mtDNA deletion transmission and age-associated dynamics and to further develop C. briggsae as an experimental model of human mtDNA deletion dynamics.

\begin{abstract}
Aim 1. Characterize nad5 $\Delta$ stability across development in C+ and C- C. briggsae natural isolates. I hypothesized that deletion levels would increase across nematode development for all isolates, but that the magnitude of this increase would be lower in isolates harboring putative compensatory mutations compared to isolates lacking those mutations.
\end{abstract}

\begin{abstract}
Aim 2. Determine the patterns of nad5 5 maternal transmission in $\mathrm{C}+$ and $\mathrm{C}-\mathrm{C}$. briggsae natural isolates. I tested the hypotheses that: 1) Deletion transmission rates would be negatively correlated with presence of compensatory mutations; i.e., greater in C- than in C+ isolates, but largely uncorrelated with parent-specific nad5 $\Delta$ levels due to mitochondrial sampling during oogenesis (Stewart and Chinnery 2015);
\end{abstract}


and 2) nad5 transmission levels would be lower in germline versus somatic tissue owing to purifying selection in the former (Clark et al. 2012; Stewart and Larsson 2014). Achievement of this aim provides the first characterization of nad5s transmission patterns and indicates whether the compensatory mutation protects offspring from deletion inheritance.

Chapter 2 reports the results of standard PCR-based and qPCR assays on samples from $\mathrm{C}+$ and C- C. briggsae natural isolates for characterization of age- and transmission-related changes in nad5s heteroplasmy. General conclusions and directions for future work are discussed in Chapter 3. 
C H A P TER 2: Accumulation and Transmission Dynamics of a Naturally-Occurring mtDNA Deletion in Caenorhabditis briggsae

B a c k ground

Mitochondria are essential organelles of nearly all eukaryotes, generating the majority of their cells' energy stores in the form of adenosine triphosphate (ATP) via the mitochondrial electron transport chain (ETC). When mutation influences the proper formation and function of the ETC components, affected organisms can experience negative physiological and fitness consequences. Mitochondrial DNA (mtDNA) is thought to be particularly susceptible to genomic decay due to deleterious mutation accumulation (Lynch et al. 1995)—a hallmark of Muller's Ratchet (Muller 1964) — owing to its uniparental inheritance, high mutation rates, and a lack of effective recombination (Muller 1964; Felsenstein 1974; Gabriel et al. 1993; Lynch et al. 2012). Human mitochondrial diseases have been linked to both point and deletion mutations in mtDNA, with single large-scale mtDNA deletions accounting for a quarter of all mitochondrial disorders present in adults (Chinnery et al. 2000; Pitceathly et al. 2012). Somatic tissues have been found to form and accumulate mtDNA deletion mutations at a higher rate than germline tissues in most studied systems (Tsang and Lemire 2002; Sato et al. 2007), but the risk of transmission of deleterious mtDNA deletions across generations-though relatively rare-remains. Importantly, mitochondria are inherited in a nearly exclusively maternal fashion; molecular mechanisms that eliminate paternal sperm mitochondria upon egg 
fertilization have been described in Caenorhabditis nematodes (Sato and Sato 2011), mice (Shitara et al. 2000) and other organisms.

Despite the important implications of mtDNA deletions for human health, the forces controlling their formation, age-related accumulation and risk of transmission remain largely unknown. Studying the dynamics of accumulation and transmission of deletion mutations in the Caenorhabditis briggsae model system will aid in elucidating the forces shaping mtDNA deletion formation and abundance in eukaryotes, and a first step toward conceiving methods by which mtDNA deletions can be manipulated and identifying potential therapies for use in treating human pathologies.

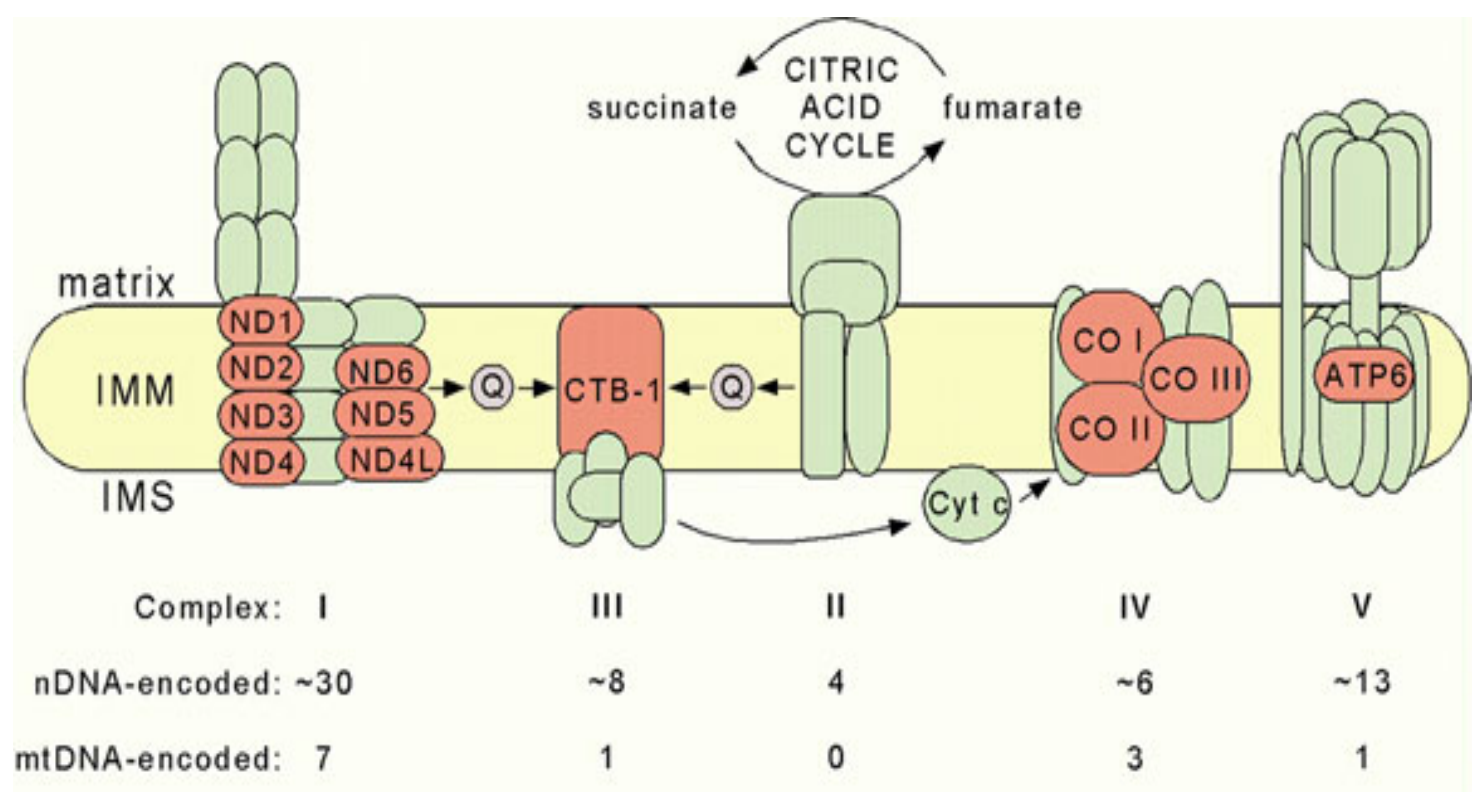

Figure 1. Schematic of Caenorhabditis mitochondrial electron transport chain (ETC). Mitochondrial-encoded components are shown in orange while subunits encoded by the nuclear genome are shown in green. Protons are pumped from the matrix side of the inner mitochondrial membrane (IMM) to the intermembrane space (IMS). $Q=$ ubiquinone; Cyt $c$ = cytochrome . . (Lemire 2005) 
Mitochondrial electron transport chain. The mitochondrial ETC is a supercomplex of five enzymatic proteins composed of subunits encoded by both the nuclear and mitochondrial genomes and is the site of oxidative phosphorylation (OXPHOS) (Figure 1). Aerobic eukaryotes depend on mitochondrial respiration and OXPHOS to produce the energy required for life; thus, mitochondria play a central role in cellular biology. During OXPHOS, electrons are passed through the series of protein complexes as hydrogen ions are pumped across the inner mitochondrial membrane. In the ETC, electrons obtained from nutrients flow down the mitochondrial inner membrane, moving from reduced to oxidized states. The process is initiated by either oxidation of NADH by complex I or succinate by complex II. Electrons are passed to coenzyme $\mathrm{Q}$ (ubiquinone) which transfers them to complex III. Cytochrome $c$ then passes them to complex IV and finally to oxygen. The energy released during this process pumps protons across the mitochondrial inner membrane and into the intermembrane space. The transmembrane potential drives complex $\mathrm{V}$ to generate ATP by combining ADP and free phosphate. Mitochondrial ATP is exported to the cytosol and this raw energy is then utilized by various cellular reactions (Wallace 2016).

Because the ETC is partially encoded by mtDNA (Figure 1), mtDNA integrity is crucial to organismal health and fitness. Essential ETC components including NADHdehydrogenase-1, cytochrome- $b$, cytochrome-c-oxidase-I, several transfer RNA (tRNA) and ribosomal RNA (rRNA), and others are mitochondrially encoded (Stewart and Chinnery 2015). Mitochondrial diseases in humans are primarily caused by dysfunction in respiration and phosphorylation processes of the ETC as a result of 
mutation, and are especially destructive to high energy-demand tissues and organs such as heart and brain. Mutations in mtDNA have been demonstrated to lead to increased electron leakage in the ETC and over-production of reactive oxygen species (ROS) within the mitochondria (Pitkanen et al. 1996).

mtDNA deletion mutations. Large-scale deletions, often spanning several genes, comprise a significant proportion of all known disease-causing mtDNA mutations in humans. Such mutations can occur sporadically in early development and remain in cell lineages, ultimately accumulating in affected tissues. In addition to sporadic formation within individuals, mtDNA mutations can also be inherited maternally (Brown et al. 2006), although inheritance of large-scale deletions appears to be less common (Poulton et al. 1991). This is perhaps due to increased levels of selection acting on deletions compared to single base mutations as well as the effects of mtDNA sampling thought to occur between generations (i.e., mitochondrial bottleneck effect; Hauswirth and Laipis 1982), and/or to oocyte-specific mtDNA stability (De Paula et al. 2013). Indeed, there are extremely few examples of heritable human diseasecausing mtDNA deletions; rather, these mutations tend to arise sporadically and multiply within somatic tissues.

MtDNA deletions generally exist in a heteroplasmic state, with a mixture of both mutant and wildtype mtDNAs present within an individual, where biochemical defects manifest beyond a certain heteroplasmy threshold (Chinnery et al. 2000; Sato et al. 2007). The amount and distribution of the deletion throughout tissues are the primary factors in determining the severity of the disease phenotype and clinical 
symptoms (Moraes et al. 1999; Vielhaber et al. 2000; Gomez-Gomez 2017), and heteroplasmy levels of disease-associated mtDNA deletions are often found to increase with age (Stewart and Chinnery 2015). These mtDNAs therefore behave as "selfish genetic elements", accumulating at the expense of their organismal host. In order to be classified as a selfish element the DNA region must be detrimental to the organism (i.e., the deletion has negative consequences at high heteroplasmy levels) and it must increase in frequency at the expense of organismal fitness (i.e., has a replicative advantage in the absence of natural selection). In deletion-bearing genomes, in particular, this effect may be due to their faster replication times, which allow them to outcompete intact genomes (Clark et al. 2012), or due to replicationdependent repair processes (Phillips et al. 2017).

The mechanism(s) by which mtDNA deletions arise is still debated (Krishnan et al. 2008), but some mtDNA deletions may occur due to existing mutations in nuclear genes involved in mtDNA maintenance and replication and mitochondrial nucleotide metabolism (Kaukonen et al. 2000; Spelbrink et al. 2001; Hudson and Chinnery 2006). Deletions frequently occur between the origins of replication on heavy and light strands, and deleted sites are often flanked by short direct repeats, pointing to potential issues during human mtDNA replication or repair (Shoffner et al. 1989; Mita et al. 1990; Samuels 2004; Krishnan et al. 2008; Reeve et al. 2008). In a study of patients with Kearns-Sayre Syndrome and related disorders, Mita et al. (1990) found that deletions with precisely flanking direct repeats were significantly more common than deletions with imprecisely positioned repeats, leading them to conclude that short direct repeats in mtDNA promote a recombination mechanism 
which eliminates intervening sequences. Importantly, the length of sequence homology plays a significant role. For instance, Albertini et al. (1982) demonstrated that the frequency of large deletion formation in Escherichia coli was reduced by an order of magnitude by altering a single base pair within a flanking repeat; however, shorter repeats with greater sequence homology were more predictive of deletion formation than longer repeats with less sequence homology (Mita et al. 1990).

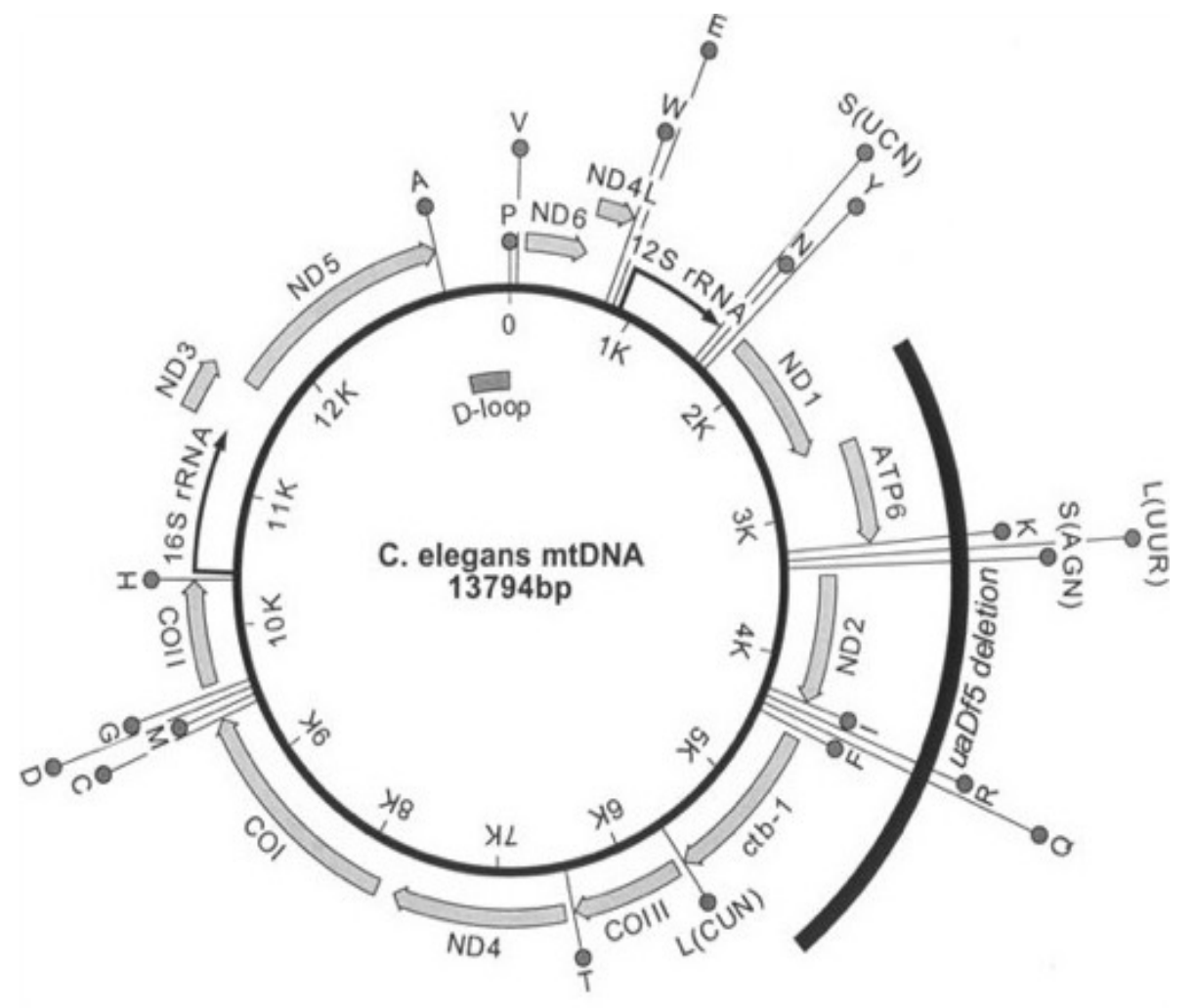

Figure 2. Mitochondrial genome map of congener species Caenorhabditis elegans showing the region affected by the uaDf5 deletion (Lemire 2005).

In humans, the 4977-bp "common" mtDNA deletion-named for its common occurrence and association with several clinical disease phenotypes-removes 4,977 basepairs between the ATPase8 and ND5 genes, and affects important gene products involved in OXPHOS. The deletion is heteroplasmic, with clinical manifestations at 
levels exceeding a critical threshold (Wallace and Chalkia 2013); it is implicated in numerous types of cancers and other disease phenotypes when present at high levels (Dani et al. 2003; Chen et al. 2011; Nie et al. 2013) and is also found to accumulate with age. It is typically associated with sporadic disease phenotypes resulting from accumulation in somatic tissues; despite this, the deletion has been discovered in unfertilized human oocytes, suggesting that it may also be frequently inherited (Hsieh et al. 2002; Chan et al. 2006). Dani et al. (2003) found that this deletion appeared to experience greater selective pressure in highly-proliferative tumor tissue, whereas tissues with lower proliferative rates tended to allow for greater accumulation of the mutation to varying degrees (Dani et al. 2003).

Because few experimental models of heritable deletions exist, most of our knowledge of such mutations relies on human pedigree studies. An exception comes from a single large-scale deletion, uaDf5 (Figure 2), present in experimental Caenorhabditis elegans nematode populations. This deletion, which removes 11 genes, was generated by mutagenesis and is present exclusively in a heteroplasmic state ranging from $\sim 20-80 \%$ per worm (Tsang and Lemire 2002; Lemire 2005). Although previously thought not to present a distinctive phenotype (Tsang and Lemire 2002), more recent analysis discovered that uaDf5 impacts metabolism, sperm performance, lifespan and fitness in affected individuals (Liau et al. 2007). Tsang and Lemire (2002) suggested that the uaDf5 deletion is maintained in laboratory populations due to two opposing forces (i.e., one that increases low uaDf5 levels and another that decreases high $u a D f 5$ levels) working in tandem. They further suggested that the deletion may not always present a phenotype because mtDNA copy 
number is roughly double in heteroplasmic individuals by comparison to their wildtype counterparts; i.e., increased mtDNA copy number could serve as a method of compensation for the energy deficit resulting from compromised organelles.

Caenorhabditis briggsae. The model nematode, Caenorhabditis briggsae, a relative of $C$. elegans, offers a favorable system for experimental study of mutation genetics within populations, including mitochondrial deletion dynamics. C. briggsae nematodes, like $C$. elegans, exhibit an androdioecious mode of reproduction, have a short generation time and lifespan, and are well-suited to laboratory culture. Unlike C. elegans, however, C. briggsae exhibits a higher rate of mutation at certain nuclear sites; is more susceptible to large mtDNA deletions (and possibly also to large mtDNA insertion events; Howe et al. 2010); and exhibits greater molecular genetic diversity and more extreme population genetic substructuring (Baer et al. 2005).

Natural populations of Caenorhabditis briggsae belong to three distinct clades: I (previously Tropical), II (previously Temperate), and III (previously Kenyan or Equatorial). Importantly, two pseudogenes evolved in the $C$. briggsae lineage, likely from inserted repeats of nad-5: Ynad5-1 which is present in all known natural isolates, and $\Psi$ nad5-2 which is only present in clade I and II isolates, and lacking in all other Caenorhabditid species tested (Figure 3). 


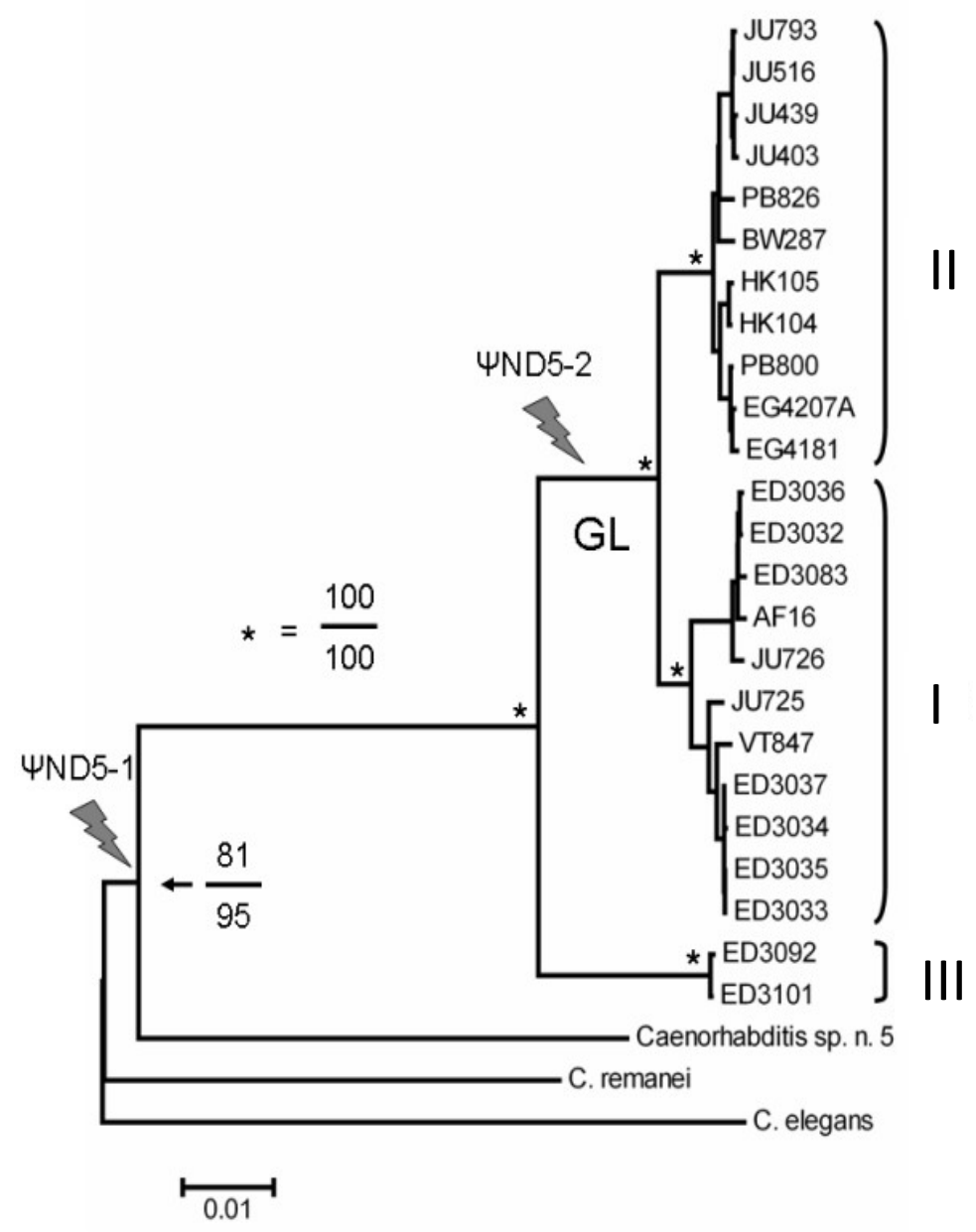

Figure 3. Evolutionary origins of $\Psi$ nad5 elements. The phylogenetic relationships between Clade II (previously Temperate), Clade I (previously Tropical) and Clade III (previously Kenyan or Equatorial) of Caenorhabditis briggsae, and other Caenorhabditid species. Lightning bolts indicate the evolution of two important pseudogene repeats in the C. briggsae genome. (Adapted from Howe and Denver 2008)

nad5 $\Delta$ formation. The placement of pseudogene $\Psi$ nad5-2 appears to promote a large, heteroplasmic mtDNA deletion, which removes the first 786 base pairs of the NADH-dehydrogenase-5 (nad5) gene and dozens of highly conserved codons (Howe and Denver 2008) (Figure 4). The normal gene product of nad5 functions as an integral component of complex I of the mitochondrial electron transport chain; 
consequently, the deletion (named nad5 5 ) is found exclusively in a heteroplasmic state (i.e., homoplasmy would be lethal). Importantly, nad5 $\Delta$ arises sporadically and is also maternally inherited, making it a good model to study scenarios of both somatic deletion accumulation and germline inheritance. The canonical nad5s, as described by Howe et al. 2010, likely arises repeatedly alongside other similar deletions affecting the same region (Phillips et al. 2015; Fig. 3). Our research group

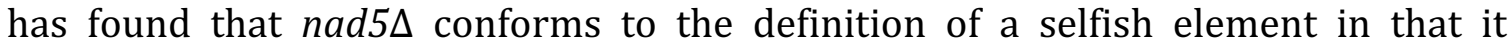
increases in frequency across nematode generations under genetic drift (Howe and

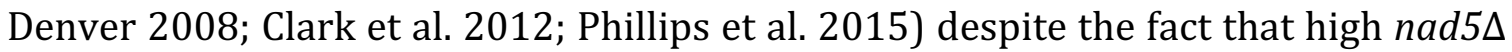
heteroplasmy levels are associated with reduced isolate-specific fecundity and other phenotypes (Estes et al. 2011). It is important to note that $C$. briggsae nad5 $\Delta$ does not appear to exhibit mtDNA copy number compensation (as observed in C. elegans uaDf5; Tsang and Lemire 2002), although this observation is based on limited data (J. Wagner, D. K. Howe, S. Estes, D. R. Denver, unpubl.). The nad5 $\Delta$ deletion exists at a maximum heteroplasmic threshold of approximately $60 \%$ within adult individuals; it is assumed that levels greater than $60 \%$ would be lethal. The nad $5 \Delta$ deletion values shown in Figure 5 represent the average percent heteroplasmy (or proportion of mtDNAs harboring the deletion) for each natural isolate examined in a previous study by Howe and Denver (2008). These particular isolates, a subset of which were selected for this project, exhibit heteroplasmy levels ranging from $0 \%$ to greater than $50 \%$. 


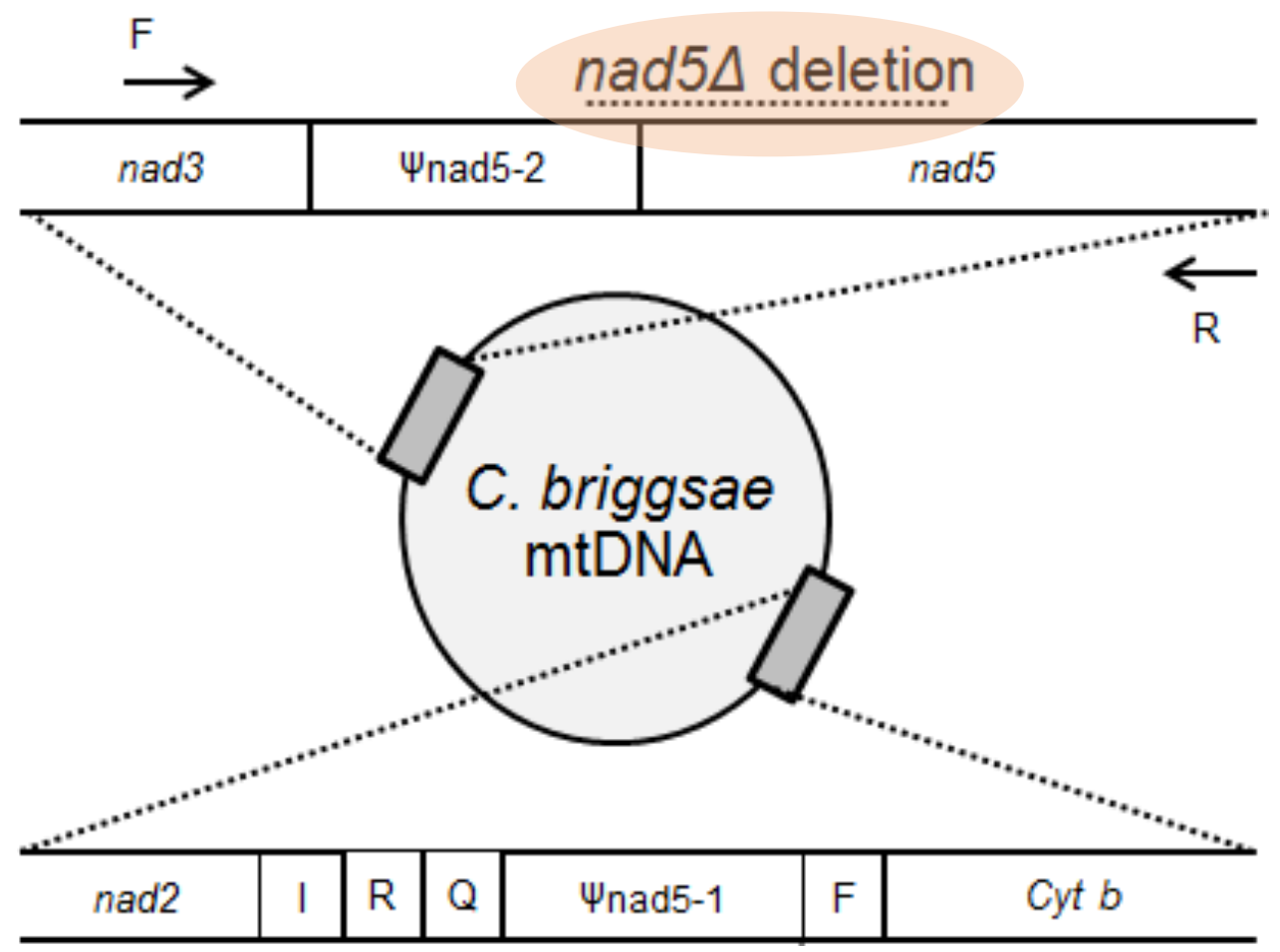

Figure 4. $\Psi$ nad5 element positions in $C$. briggsae mitochondrial genomes. Flanking repeats of pseudogene $\Psi$ nad5-2 likely cause mismatch during replication, promoting a large heteroplasmic deletion mutation, nad5 (indicated by orange shaded area) (Adapted from Howe and Denver 2008).

Putative compensatory mutation. nad5s is thought to repeatedly form during DNA replication as a result of mispairing between repeats flanking $\Psi$ nad5-2 and nad5 (Figure 4), meaning that the deletion can accumulate due to both inheritance and to de novo formation from a previously intact genome. Deletion formation is likely a result of mispairing or recombination rather than replication-dependent repair; $C$. elegans (and most likely C. briggsae) mitochondrial genomes replicate by an ancestral rolling circle mechanism (Chen 2013; Lewis et al. 2015). Importantly, certain $C$. briggsae isolates contain putative compensatory mutations $(\mathrm{C}+)$ that reduce the similarity of the flanking repeats, and are hypothesized to limit nad5 $\Delta$-formation 


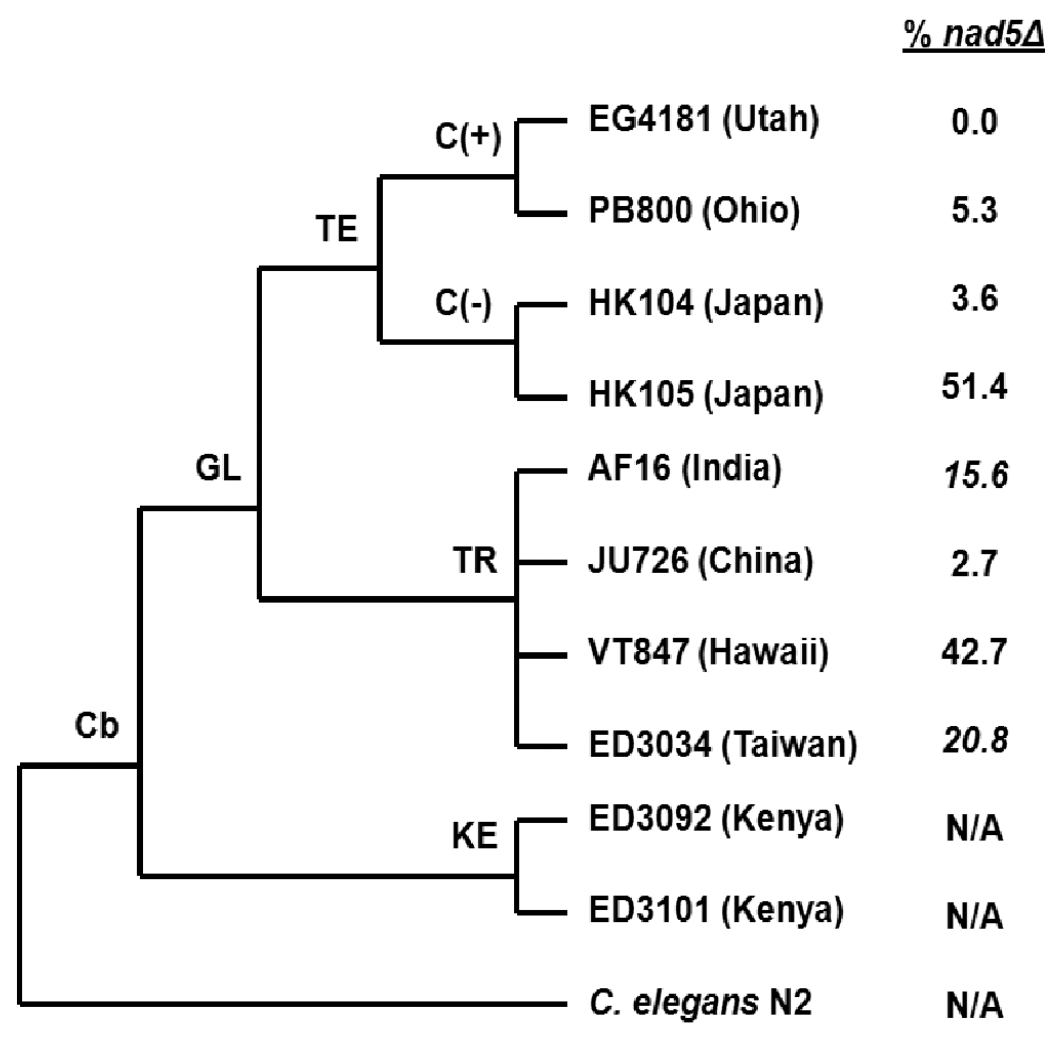

Figure 5. Phylogenetic relationships and nad5 $\Delta$ heteroplasmy levels of $C$. briggsae isolates. nad5 5 heteroplasmy levels are variable in natural populations of Caenorhabditis briggsae, ranging from $0 \%$ deletion to nearly $60 \%$ deletion. Putative compensatory mutations $(\mathrm{C}+$ ) exist in a small subset of $C$. briggsae isolates (including EG4181 and PB800) and may aid in protecting these populations from nad5 $\Delta$ deletion formation. $G L=$ global superclade including clades I and II; TE = clade II (previously Temperate); TR = clade I (previously Tropical); KE = clade III (previously Kenyan or Equatorial). (Hicks et al. 2012)

events compared to isolates lacking these mutations (C-) (Figure 6). Specifically, a more recently diverged group within clade II, which includes natural isolates EG4181 and PB800, has evolved such mutations within the flanking repeats (Howe and Denver 2008; Fig. 4). Consistent with this idea, C+ isolates exhibit lower overall nad5s accumulation across multiple generations of evolution under genetic drift conditions as compared to C- isolates (Clark et al. 2012; Phillips et al. 2015). However, it remains unclear how nad5s frequency changes across a single generation (during 
transmission from mothers to offspring) or with nematode age, and if the presence of compensatory mutations influences these patterns.

Despite the importance of understanding mtDNA deletion dynamics for human health, the forces influencing their formation, age-related accumulation and risk of transmission remain understudied. This project explored the dynamics of mtDNA deletion accumulation across development of an individual, and the effects of mtDNA deletion transmission from parent to offspring. The latter was achieved by

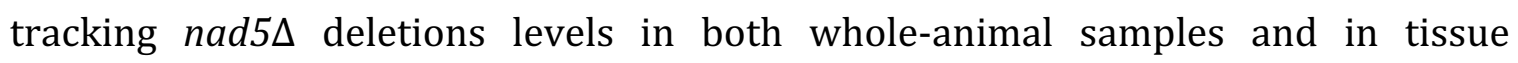
dissections to discern patterns of heteroplasmy in somatic versus germline tissues. Both primary foci of the thesis project (i.e., developmental accumulation and generational transmission) assessed the effects of putative compensatory mutation on deletion dynamics by utilizing specific natural isolates that do or do not contain compensatory mutations. 


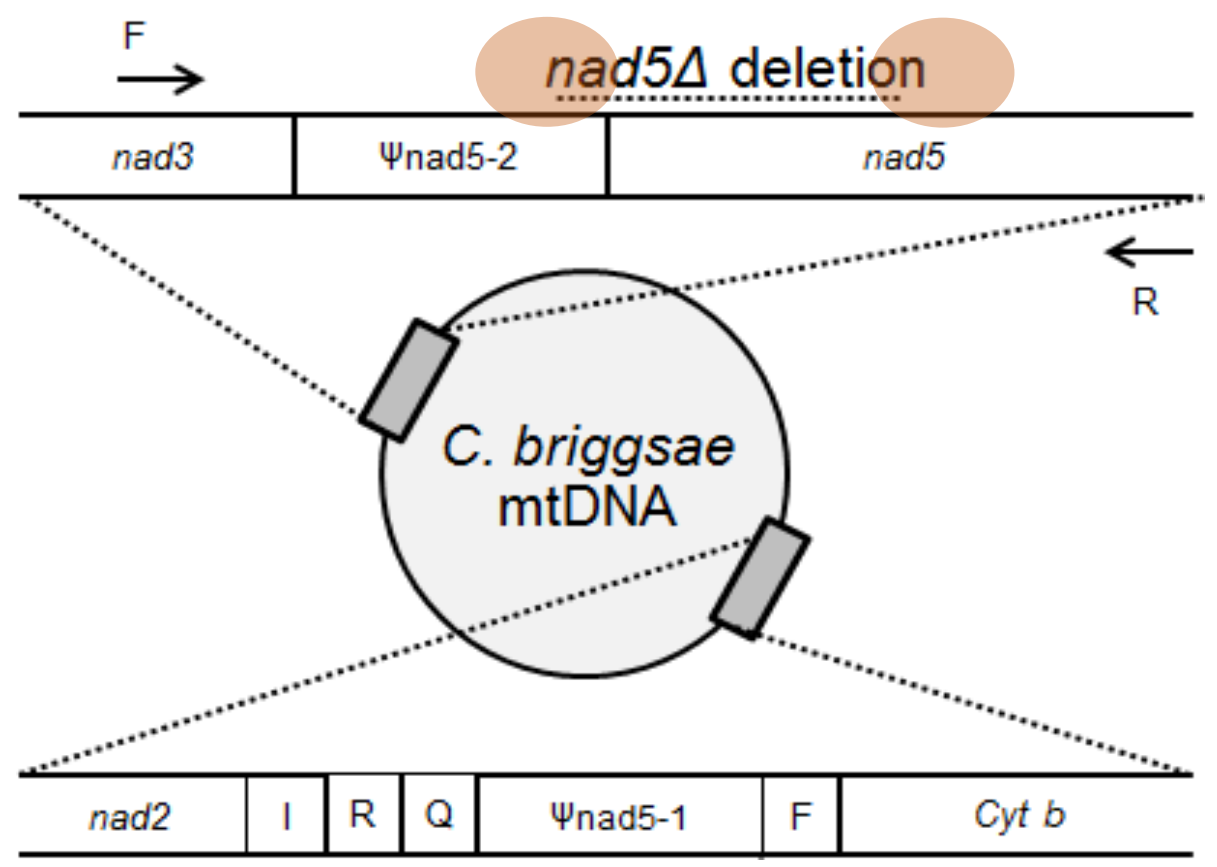

Figure 6. Sequence divergence in flanking regions may confer compensatory protection. Placement of putative compensatory mutations in the form of additional sequence divergence (indicated by orange shaded areas) help to protect against new deletion formation. (Adapted from Howe and Denver 2008) 
M e thods

Nematode strains and whole-worm sampling. This thesis utilized five natural isolates of Caenorhabditis briggsae (Table 1; Figure 7A), all of which were obtained from the Charles Baer lab at University of Florida (Gainesville, FL, USA) and/or the Dee Denver lab at Oregon State University (Corvallis, OR, USA). These isolates include: AF16, ED3034, EG4181, HK105, and PB800, selected for their natural variation in nad5 $\Delta$ heteroplasmy levels, with PB800 and EG4181 specifically selected due to their putative compensatory mutations described below. HK105 was chosen because this isolate was noted as having an especially high nad5 $\Delta$ level by Howe and Denver (2008). It is unknown why the average deletion level of this isolate differs so dramatically from that of HK104, another Japanese isolate (Figure 5). The divergence may have occurred during laboratory propagation; however, Howe and Denver (2008) suggested that the difference may have arisen via genetic drift if these island isolates were recently established from very small populations. It is notable that the other island isolate, VT847 from Hawaii, also exhibited an exceptionally high nad5 $\Delta$ heteroplasmy level (Figure 5). In summary, the source(s) of natural nad5 $\Delta$ heteroplasmy variation among the isolates utilized in this study is unknown, but it is likely that these differences are defined by among-isolate differences in rates of deletion formation and relative strengths of genetic drift and a number of selective forces.

Stocks were thawed and maintained on $60-\mathrm{mm}$ plates containing Nematode Growth Medium Lite (NGM-Lite; US Biological Life Sciences) medium treated with 
$200 \mathrm{mg} / \mathrm{ml}$ streptomycin sulfate (Sigma-Aldrich), with a lawn of strain OP50-1

Escherichia coli as the sole food source, and kept in constant incubation at $20^{\circ} \mathrm{C}$. All strains were allowed to recover from freezing for two to three generations prior to sampling, and were transferred by "chunking" before picking isolated individuals to new plates.

Table 1. Caenorhabditis briggsae natural isolates used for this study. C. briggsae natural isolates, phylogenetic clades, geographical origins, and associated average $\%$ nad5 $\Delta$ heteroplasmy as found by the Denver lab (Howe and Denver 2008) and reported in Estes et al. 2011. These numbers are based on very small sample sizes.

\begin{tabular}{|c|c|c|c|}
\hline Isolate & Clade & Origin & Average \% nad5 \\
\hline AF16 & I & Ahmedabad, India & 15.6 \\
\hline ED3034 & I & Taipei, Taiwan & 20.8 \\
\hline EG4181 & II & Salt Lake City, Utah, USA & 0.0 \\
\hline HK105 & II & Sendai, Japan & 51.4 \\
\hline PB800 & II & Dayton, OH, USA & 5.3 \\
\hline
\end{tabular}

For standard PCR analysis in whole worms, individual nematodes of AF16, ED3034, HK105, and PB800 at each major developmental stage including L1 (first larval), L2, L3, L4 and Young Adult (target $n=64$ per life stage per isolate for a total of 320 sampled individuals) were picked into $15 \mu$ of worm lysis buffer (containing $50 \mathrm{mM} \mathrm{KCl}, 2.5 \mathrm{mM} \mathrm{MgCl}_{2}, 10 \mathrm{mM}$ Tris, $0.45 \%$ Tween 20, 0.05\% gelatin, and $60 \mu \mathrm{g} / \mathrm{mL}$ Proteinase $\mathrm{K}$ in DNase- and RNase-free $\mathrm{H}_{2} \mathrm{O}$ ) and stored at $-80{ }^{\circ} \mathrm{C}$ in PCR strip tubes prior to DNA extraction (Figure 8A). Additional samples of ten reproductive 
hermaphrodites of each isolate and 16 each of their respective L1 offspring were collected via the same method (Figure 8B). These samples were collected in 2012 by a previous lab member and stored at $-80{ }^{\circ} \mathrm{C}$ until DNA was extracted and PCR reactions were performed for this study.
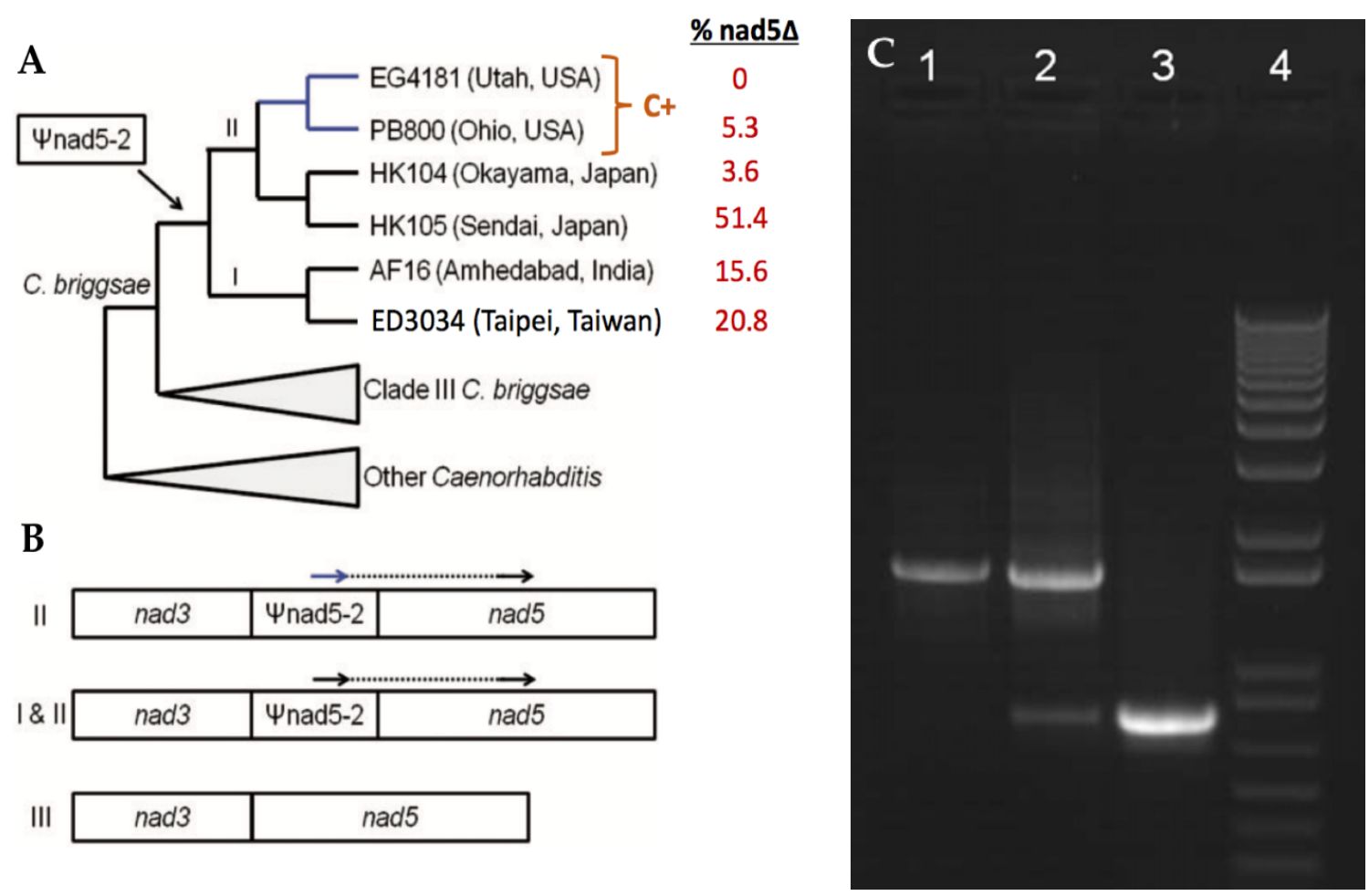

Figure 7. Phylogenetic relationships of $C$. briggsae natural isolates based on mtDNA, nad5 $\Delta$ locus schematic, and conventional PCR banding possibilities for this region. A) nad5 $\Delta$ heteroplasmy levels are variable in natural populations of Caenorhabditis briggsae, ranging from $0 \%$ deletion to nearly $60 \%$ deletion. Putative compensatory mutations $(\mathrm{C}+)$ exist in a small subset of $C$. briggsae species and may aid in protecting these strains from nad5 $\Delta$ deletion formation. B) Insertion of pseudogene $\Psi$ nad5-2 in Clades I and II C. briggsae promotes the formation of nad5 $\Delta$ between the nad 3 and nad5 regions (indicated by arrows); Clade III C. briggsae do not form nad5 $\Delta$ because the clade lacks $\Psi$ nad5-2. C) Example of possible gel banding patterns analyzed on two sets each of multi-worm lysates of AF16, ED3034, and PB800, and one set each of HK105 and EG4181. Scoring: $1=$ one heavy band present (wildtype only); 2 = two bands present (heteroplasmy); 3 = one light band present (deletion only). (Adapted from Clark et al. 2012)

An additional 20 reproductive hermaphrodites from AF16 and PB800 and ten from ED3034 and EG4181 (selected specifically to represent a second C+ isolate) and 
16 of each hermaphrodite's respective L1 offspring were collected individually into $15 \mu \mathrm{l}$ of worm lysis buffer in PCR strip tubes and cryogenically stored at $-80^{\circ} \mathrm{C}$ prior to DNA extraction, serving as whole-worm samples for both standard PCR assays for all four isolates, and qPCR assays for AF16 and PB800. Hermaphrodites at L4 stage were picked individually at random from large populations to new plates, allowed to lay eggs for $12 \mathrm{hrs}$, then transferred again to new plates. $12 \mathrm{hrs}$ later, moms and hatched L1 offspring were picked individually into worm lysis buffer. All sets of parent-L1 offspring samples were analyzed together for both PCR and qPCR assays.
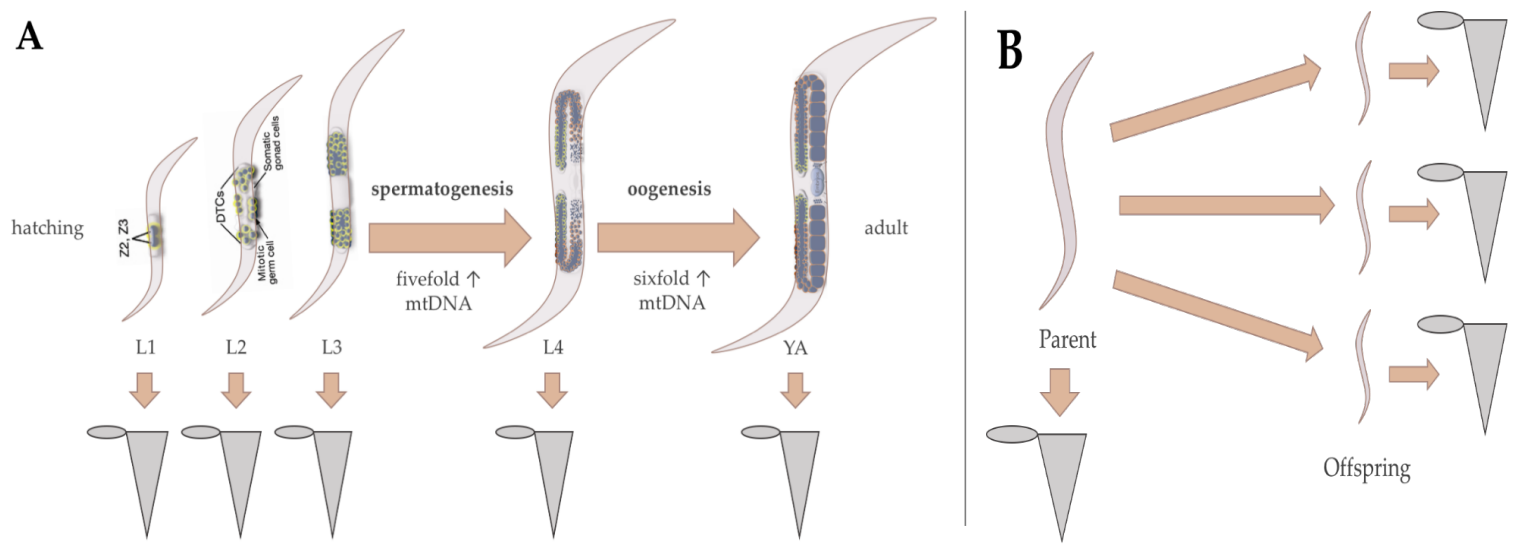

Figure 8. Sampling methods for individual worm collection prior to PCR and qPCR assays. A) Sampling methods for whole-worm PCR samples representing development from larval L1 through Young Adult (YA). Spermatogenesis between L3 and L4 and oogenesis between L4 and YA involve significant proliferations of mtDNA and has the potential to result in a dramatic change in nad5 5 proportion from L3 to YA if deleted genomes are permitted to accumulate selfishly and purifying selection is not present. B) Sampling methods for wholeworm samples representing hermaphrodite-to-offspring inheritance.

DNA extraction. For all samples, DNA was extracted via a freeze-thaw procedure to break the worm cuticle followed by processing in a Thermo Electron Corporation Px2 Thermal Cycler with the following extraction program: Incubate at $60^{\circ} \mathrm{C}$ for 1.5 hours, heat to $95{ }^{\circ} \mathrm{C}$ for 15 minutes to destroy the Proteinase $\mathrm{K}$ present in the lysis buffer, 
and cool to a holding step of $10{ }^{\circ} \mathrm{C}$. Samples were stored at $-80^{\circ} \mathrm{C}$ post-extraction and prior to PCR and qPCR assays.

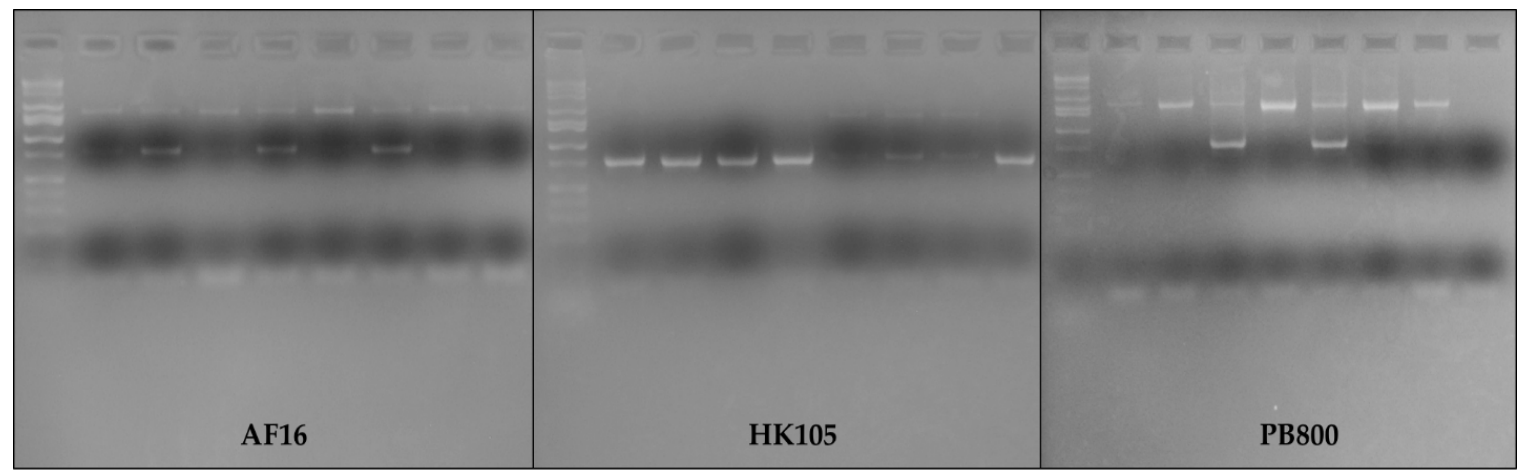

Figure 9. Representative images of gel banding patterns from whole-worm PCR reactions. AF16 demonstrates a significant proportion of scores of 2 (heteroplasmic), HK105 a significant proportion of 3 (deletion only), and PB800 a significant proportion of 1 (intact only).

PCR analysis of whole-worm nad5s abundance. Standard PCR reactions were performed on all sets of whole-animal samples of five $C$. briggsae natural isolates: AF16, ED3034, EG4181, HK105, and PB800. $3 \mu$ of undiluted template DNA was used per $50 \mu \mathrm{l}$ PCR reaction. All reactions contained $32.5 \mu \mathrm{l}$ of molecular grade DNase- and RNase-free $\mathrm{H}_{2} \mathrm{O}, 10$ ul of 5X BioLine MyTaq Reaction Buffer, $2 \mu \mathrm{l}$ each of forward and reverse primers (diluted to $10 \mu \mathrm{M}$ ), and $0.5 \mu$ BioLine MyTaq DNA Polymerase. Primer sets targeted a single region in the nad5 gene, generating a possibility of two differently sized bands, indicating presence or absence of the canonical deletion (Figure 2 in Clark et al. 2012).

PCR reactions were processed in a Thermo Electron Corporation Px2 Thermal Cycler, with the PCR reaction profile beginning with an initial denaturation step at 95 ${ }^{\circ} \mathrm{C}$ for 2 minutes; passing through 36 cycles involving denaturation at $95{ }^{\circ} \mathrm{C}$ for 30 seconds, primer annealing at $52{ }^{\circ} \mathrm{C}$ for 20 seconds, and sequence extension at $72{ }^{\circ} \mathrm{C}$ 
for 2 minutes; before finishing with a final extension step at $72{ }^{\circ} \mathrm{C}$ for 10 minutes and holding at a constant temperature of $10{ }^{\circ} \mathrm{C}$. PCR products were electrophoresed on standard $1.5 \%$ agarose gels and digitally recorded. Gel images were manually scored according to three observed banding categories, following the methods of Howe and Denver (2008), in which 1 = intact (large band only), 2 = heteroplasmic (both bands), and 3 = deletion (small band only) (Figure 7C). These manually-scored banding patterns were previously correlated with estimated deletion-bearing genome proportions obtained from qPCR results by Howe and Denver (2008) and reiterated in Clark et al. (2012), indicating that standard PCR using these primer sets can effectively serve to estimate relative genome proportions in this region.

PCR primers used in this assay included Cb_mt1F positioned in the nad3 gene, and either 58R or 36R in the nad5 gene that flank the Inad5-2 pseudogene element and its associated deletion products (if present). The three standard PCR primers used were developed for use in previous studies and provided by the Dee Denver lab at Oregon State University:

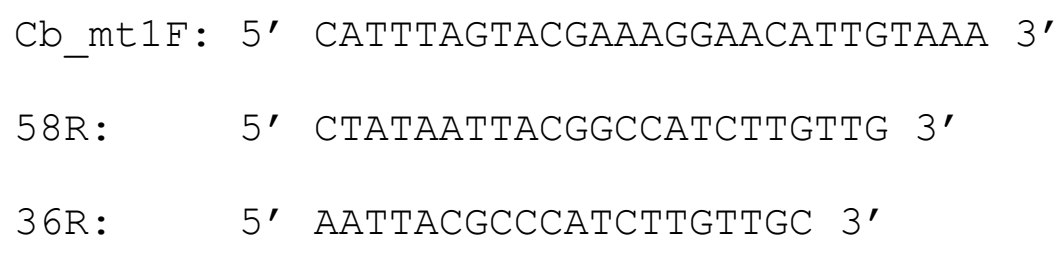

AF16, ED3034, EG4181, and HK105 all used Cb_mt1F forward primer with the 58R reverse primer, while $\mathrm{PB} 800$ required the use of $\mathrm{Cb} \_\mathrm{mt} 1 \mathrm{~F}$ with the $36 \mathrm{R}$ reverse primer due to subtle sequence variation within that region. Figure 9 shows representative examples of the gel banding produced by selected isolates of interest: 
AF16 (intermediate deletion heteroplasmy), HK105 (high deletion heteroplasmy), and PB800 (C+; low deletion heteroplasmy).

qPCR analysis of whole-worm nad5 abundance. Quantitative real-time PCR was employed on the second set of parent-L1 offspring samples to evaluate relative abundance of nad5 in AF16 and PB800 individuals. DNA was extracted as described above, quantified within each sample using a Thermo Scientific NanoDrop 2000 Spectrophotometer, then diluted to $1 \mathrm{ng} / \mu \mathrm{l} .5 \mu \mathrm{l}$ of the diluted template DNA was added to each $20 \mu \mathrm{l}$ total volume reaction. All reactions also contained $3.4 \mu \mathrm{l}$ molecular grade DNase- and RNase-free $\mathrm{H}_{2} \mathrm{O}, 10 \mu$ l Bio-Rad SsoAdvanced SYBR Green Supermix with ROX, and $0.8 \mu \mathrm{l}$ each of the appropriate forward and reverse primers each diluted to $5 \mu \mathrm{M}$.

Each sample was assayed in duplicate using two distinct primer sets. One set of qPCR primers amplified a nad5 gene region present only in intact genomes (located at the $5^{\prime}$ end of the gene) and a second set amplified a $16 \mathrm{~S}$ ribosomal RNA gene region present in both deletion-bearing and intact genomes for which there is no evidence of heteroplasmy (Howe and Denver 2008). The four qPCR primer sequences used were developed for use in previous studies and provided by the Dee Denver lab at Oregon State University:

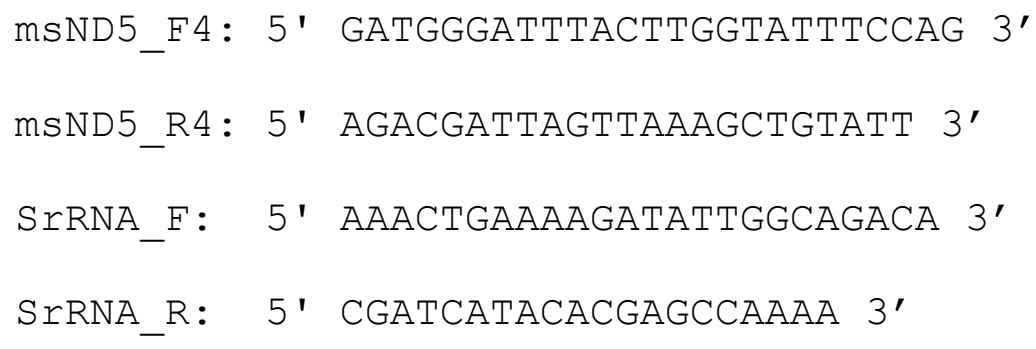


All qPCR reactions were performed in an Agilent Technologies Stratagene Mx3005P machine equipped with Agilent MxPro software for real-time qPCR data collection, using the 'Quantitative PCR' experiment type. The qPCR reaction profile began with an initial denaturation step of $95^{\circ} \mathrm{C}$ for 2 minutes; followed by 40 cycles of denaturing at $95{ }^{\circ} \mathrm{C}$ for 1 minute, primer annealing at $60{ }^{\circ} \mathrm{C}$ for 30 seconds, and sequence extension at $70{ }^{\circ} \mathrm{C}$ for 35 seconds, followed by the standard default melt curve built into the MxPro software. Amplification plots were obtained (Figure 10), displaying non-baseline-corrected fluorescence (Rn) by cycle number.

A standard curve assay was conducted using known concentrations of template DNA (provided in nanograms) prepared from a whole single-worm AF16 hermaphrodite parent and its offspring. Two separate serial dilutions were prepared from each sample type and combined individually with the two primer sets to generate four independent standard curves. Each of four serial dilutions $(25 \mathrm{ng}, 5 \mathrm{ng}$, $2.5 \mathrm{ng}, 1.25 \mathrm{ng}$ ) was processed in duplicate reactions and averaged within the Agilent MxPro software.

For sample assays, each hermaphrodite parent and its corresponding offspring were assayed together on a single plate with wells labeled in MxPro according to sample name and primer set; i.e., "mom-rRNA," "mom-nad5," "offspringrRNA," and "offspring-nad5." In total, ten plates per isolate were assayed. The 'Multiple Experiment Analysis' feature of MxPro was employed to obtain initial template DNA concentrations for each sample by comparing it to the appropriate standard curve. Each experiment (e.g., mom-rRNA) was opened in conjunction with the standard curves and baseline fluorescence thresholds were calculated separately 
for each individual experiment using the built-in feature of MxPro. Concentrations of starting template DNA were reported in nanograms, and textual data files were exported to MS Excel for further manual data processing and statistical analysis.

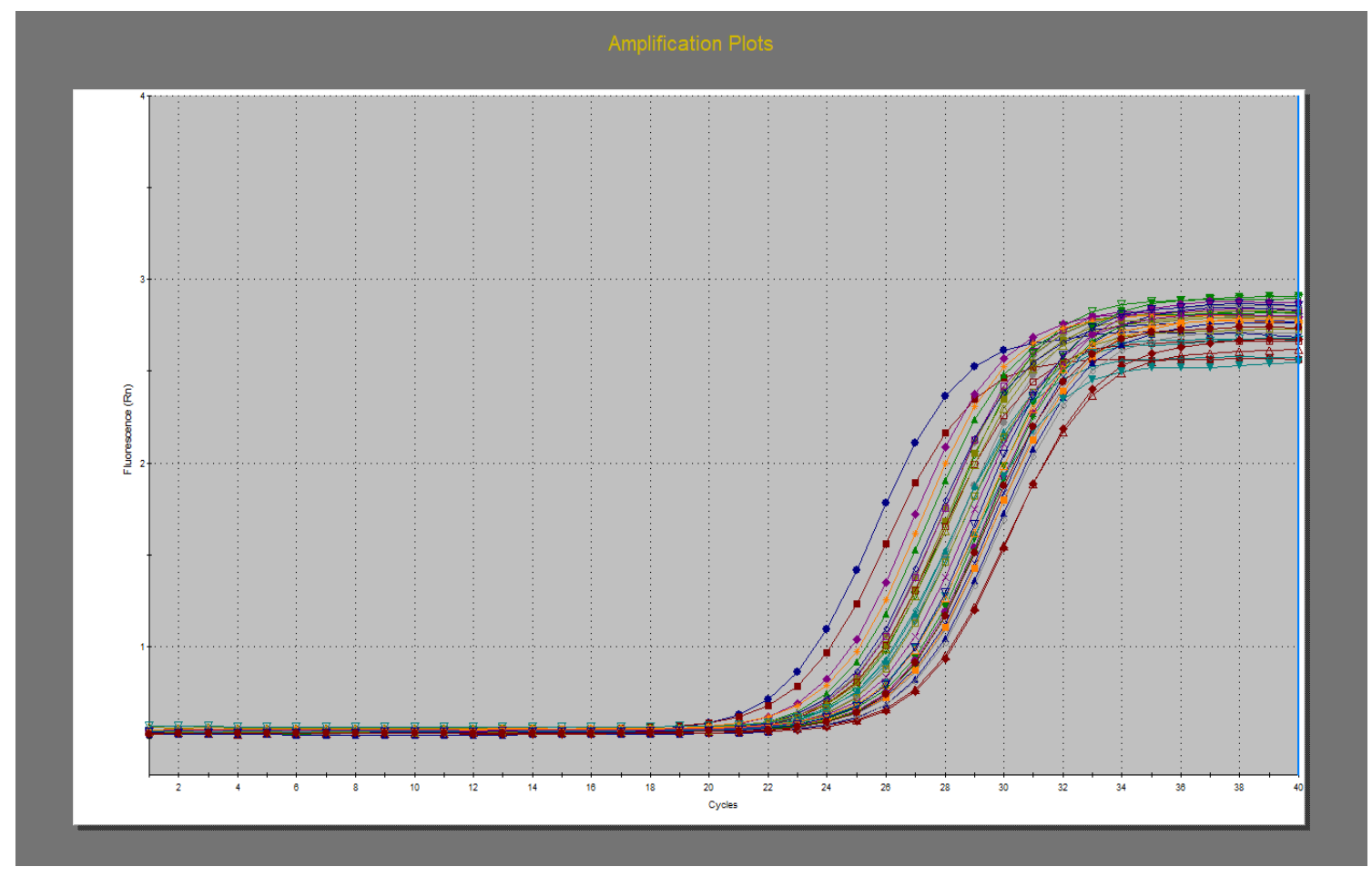

Figure 10. Representative example of amplification plots from one qPCR assay. A single plate of whole-worm unknown samples (parent and corresponding offspring), with number of cycles across the bottom axis and non-baseline-corrected fluorescence $(\mathrm{Rn})$ on the vertical axis.

\section{Gonadal dissection and qPCR analysis of somatic and germline nad5}

abundance. Following the methods of the Tim Schedl lab at Washington University (St. Louis, Missouri, USA), reproductive hermaphrodites were dissected after laying eggs for 12 hours using two sterile 18-gauge hypodermic syringe needles to cut the worm beneath the head, allowing the gonads to extrude. This treatment was applied 
to five reproductive hermaphrodites from both PB800 and AF16 isolates, and samples of gonadal and somatic (worm anterior region including the pharynx) tissues were collected separately. Additionally, eight of these hermaphrodite's respective L1 offspring were picked individually into $15 \mu \mathrm{l}$ of worm lysis buffer and cryogenically frozen in PCR strip tubes prior to DNA extraction, which was conducted as previously described. In total, ten plates containing samples from a single hermaphrodite and its offspring were assayed using the previously described qPCR methods.

Data processing and analysis. Band scoring data from all whole-worm standard PCR reactions were compiled and analyzed in JMP13 (SAS). For assays of nad5 level across stages of nematode development, a nominal logistic regression was performed to test the model: banding pattern $=\mu+$ strain + life stage $+($ strain $\mathrm{x}$ life stage $)+\varepsilon$.

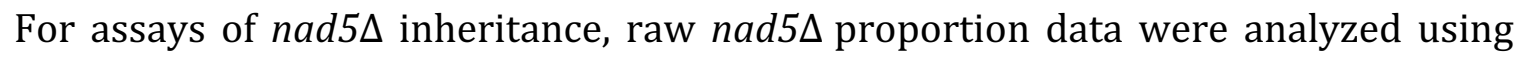
least-squares linear regressions.

For data resulting from qPCR analyses, starting template DNA concentration data from the two technical replicates for each sample-primer pair were averaged. Results from the region of interest (nad5) were divided by those from the housekeeping gene (16S rRNA) to determine the proportion of intact nad5-bearing mtDNA in each sample. This proportion was then subtracted from 1 to determine the proportion of deletion-bearing (nad5s) mtDNA. Extreme outliers resulting from failed amplification curves were removed from the data set and all negative values were converted to zero. Bivariate plots were constructed to visualize average 


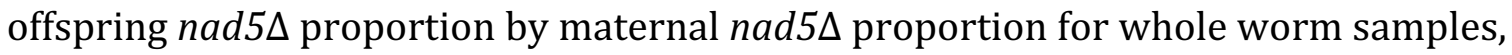
and by maternal tissue type for dissected samples. Least-squares linear regression

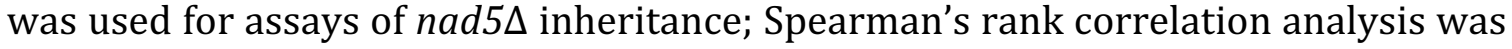
used for comparisons of nad5 levels between maternal tissue types. 
R e s u l t s

nad5s proportions increase across nematode development. Final sample sizes for the gel banding assays are provided in Table 2. Certain sample groups (e.g., PB800 L2 samples) experienced more failed PCRs than others. Results show that nad5A proportions increased across development for all isolates and that the overall nad5 $\Delta$ proportions depended upon isolate-specific starting levels (Figure 11A). The L1-stage starting levels are consistent with those previously reported (Howe and Denver 2008; Clark et al. 2012). Variance in banding pattern was primarily explained by isolate and, to a lesser extent, by life stage (Figure 11B). Comparing banding patterns between L1 and young adult samples, all isolates except for C+ PB800 exhibited statistically significant increases in deletion-bearing genomes (Cochran Armitage exact tests; $p<0.001$ ). For instance, HK105 animals exhibited a mixture of banding types at L1, which became dominated by deletion-bearing types during later stages of development, while the compensatory mutation-bearing PB800 isolate's nad5s levels were undetectable at L1 and increased slowly across development compared to other isolates. Only AF16 shows a marked change (increase) in nad5s heteroplasmy between L3 and L4, coinciding with the major mitochondrial expansion during the development of the germline. This was followed by a slight decrease in young adult worms that occurred in both AF16 and, to a lesser extent, in HK105. 
Table 2. PCR banding by isolate by life stage. Average band value reports average of band scores 1, 2, and 3 per isolate per life stage across development. C+ and Cdesignations denote whether or not the isolate contains a putative compensatory mutation. 1 S.E.M. value reported. $n$ = final sample size of successful PCR reactions per group.

\begin{tabular}{|c|c|c|c|c|}
\hline Isolate & Life stage & Average band value & S.E.M. & $n$ \\
\hline \multirow{5}{*}{ 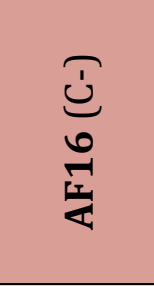 } & L1 & 1.5000 & 0.0944 & 54 \\
\hline & $\mathrm{L} 2$ & 1.3636 & 0.0704 & 55 \\
\hline & L3 & 1.7969 & 0.0597 & 64 \\
\hline & $\mathrm{L} 4$ & 1.9063 & 0.0484 & 64 \\
\hline & Young Adult & 1.5000 & 0.0704 & 64 \\
\hline \multirow{5}{*}{ 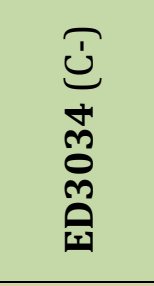 } & L1 & 1.1754 & 0.0508 & 57 \\
\hline & $\mathrm{L} 2$ & 1.4032 & 0.0628 & 62 \\
\hline & L3 & 1.4828 & 0.0662 & 58 \\
\hline & $\mathrm{L} 4$ & 1.5167 & 0.0651 & 60 \\
\hline & Young Adult & 1.5000 & 0.0630 & 64 \\
\hline \multirow{5}{*}{ 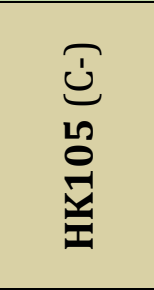 } & L1 & 2.5000 & 0.1002 & 44 \\
\hline & $\mathrm{L} 2$ & 2.9800 & 0.0200 & 50 \\
\hline & L3 & 2.9375 & 0.0378 & 64 \\
\hline & $\mathrm{L} 4$ & 2.8125 & 0.0492 & 64 \\
\hline & Young Adult & 2.9375 & 0.0305 & 64 \\
\hline \multirow{5}{*}{ 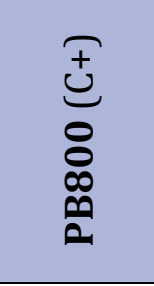 } & L1 & 1.0000 & 0.0000 & 45 \\
\hline & $\mathrm{L} 2$ & 1.2105 & 0.1228 & 19 \\
\hline & L3 & 1.1351 & 0.0791 & 37 \\
\hline & L4 & 1.2453 & 0.0655 & 53 \\
\hline & Young Adult & 1.3333 & 0.0640 & 63 \\
\hline
\end{tabular}



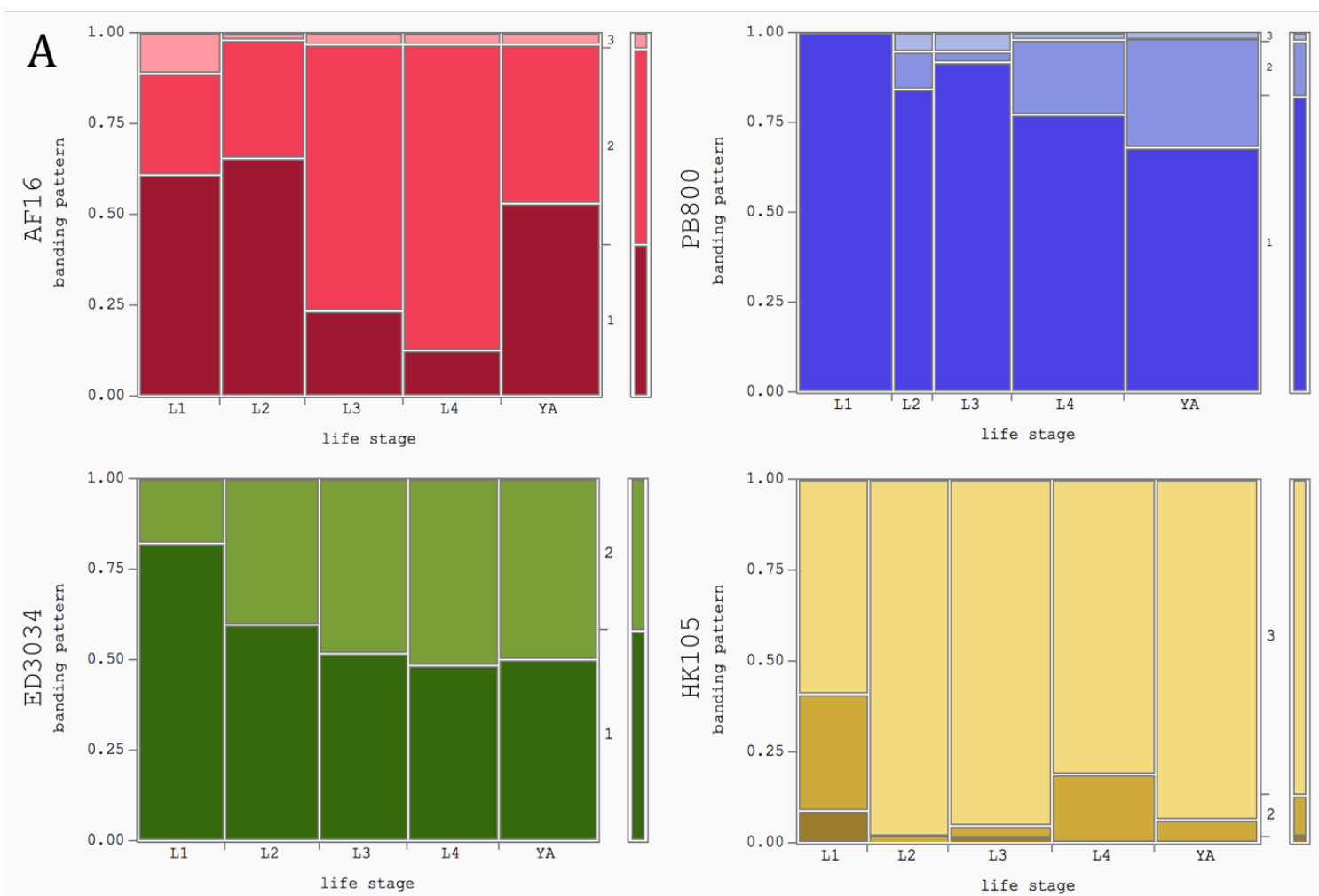

B Effect Likelihood Ratio Tests

\begin{tabular}{|c|c|c|c|c|}
\hline Source & Nparm & DF & $\begin{array}{r}\text { L-R } \\
\text { ChiSquare }\end{array}$ & Prob $>$ ChiSq \\
\hline Strain & 13 & 3 & 978.064485 & $<.0001$ * \\
\hline Life stage & 4 & 4 & 70.629603 & $<.0001 *$ \\
\hline Strain*Life stage & 12 & 12 & 64.8382405 & $<.0001 *$ \\
\hline
\end{tabular}

Figure 11. Banding score proportions by life stage by isolate and effect likelihood ratio tests. A) Proportion of each banding score across developmental life stage from L1 to Young Adult for four representative Clade I and II isolates. Darkest shades represent a score of 1 (intact only), medium shades represent a score of 2 (heteroplasmic), and lightest shades indicate a score of 3 (deletion only). Width of column represents relative sample size across life stages. B) Effect likelihood ratio tests of the above data. 
Table 3. PCR banding by isolate by inheritance. Average band value reports average of band scores 1,2, and 3 per isolate per life stage across inheritance. $\mathrm{C}+$ and $\mathrm{C}$ - designations denote whether or not the isolate contains a putative compensatory mutation. 1 S.E.M. value reported. $n=$ final sample size of successful PCR reactions per group.

\begin{tabular}{|c|c|c|c|c|}
\hline Isolate & Life stage & Average band value & S.E.M. & $n$ \\
\hline \multirow{2}{*}{ 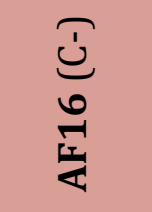 } & Parent & 1.5000 & 0.1147 & 20 \\
\hline & Offspring & 1.6809 & 0.0386 & 257 \\
\hline \multirow{2}{*}{ 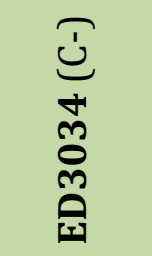 } & Parent & 1.5714 & 0.2020 & 7 \\
\hline & Offspring & 1.3684 & 0.1137 & 19 \\
\hline \multirow{2}{*}{ 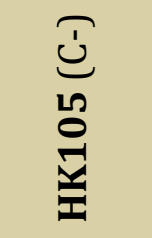 } & Parent & 2.9000 & 0.1000 & 10 \\
\hline & Offspring & 2.8471 & 0.0288 & 157 \\
\hline \multirow{2}{*}{ 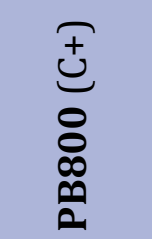 } & Parent & 1.5000 & 0.1147 & 20 \\
\hline & Offspring & 1.0000 & 0.0000 & 181 \\
\hline
\end{tabular}

Compensatory mutation reduces nad5 transmission. Final sample sizes for the transmission gel banding assays are provided in Table 3. Once again, certain sample groups (e.g., ED3034 offspring samples) experienced more failed PCRS than others. The gel banding results from parent-offspring whole-worm samples demonstrated

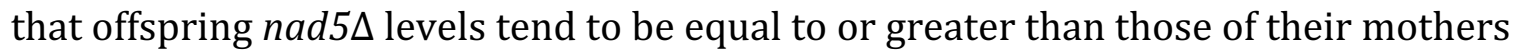
(Figure 12). Again, PB800 exhibited lower deletion levels, and also the lowest rates of nad5 $\Delta$ offspring transmission (i.e., the highest rates of reversions to wildtype banding 
pattern in offspring) than isolates that do not contain compensatory mutations. Indeed, all PB800 parents with heteroplasmic banding type 2 only produced offspring with wildtype banding type 1 . Conversely, parents of the lowest nad5s banding level categories measured for the other isolates always produced a fraction of offspring

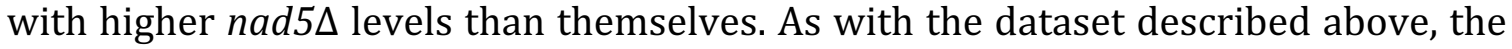
baseline nad5 $\Delta$ levels measured for each isolate generally agreed with previous findings (Howe and Denver 2008; Clark et al. 2012).

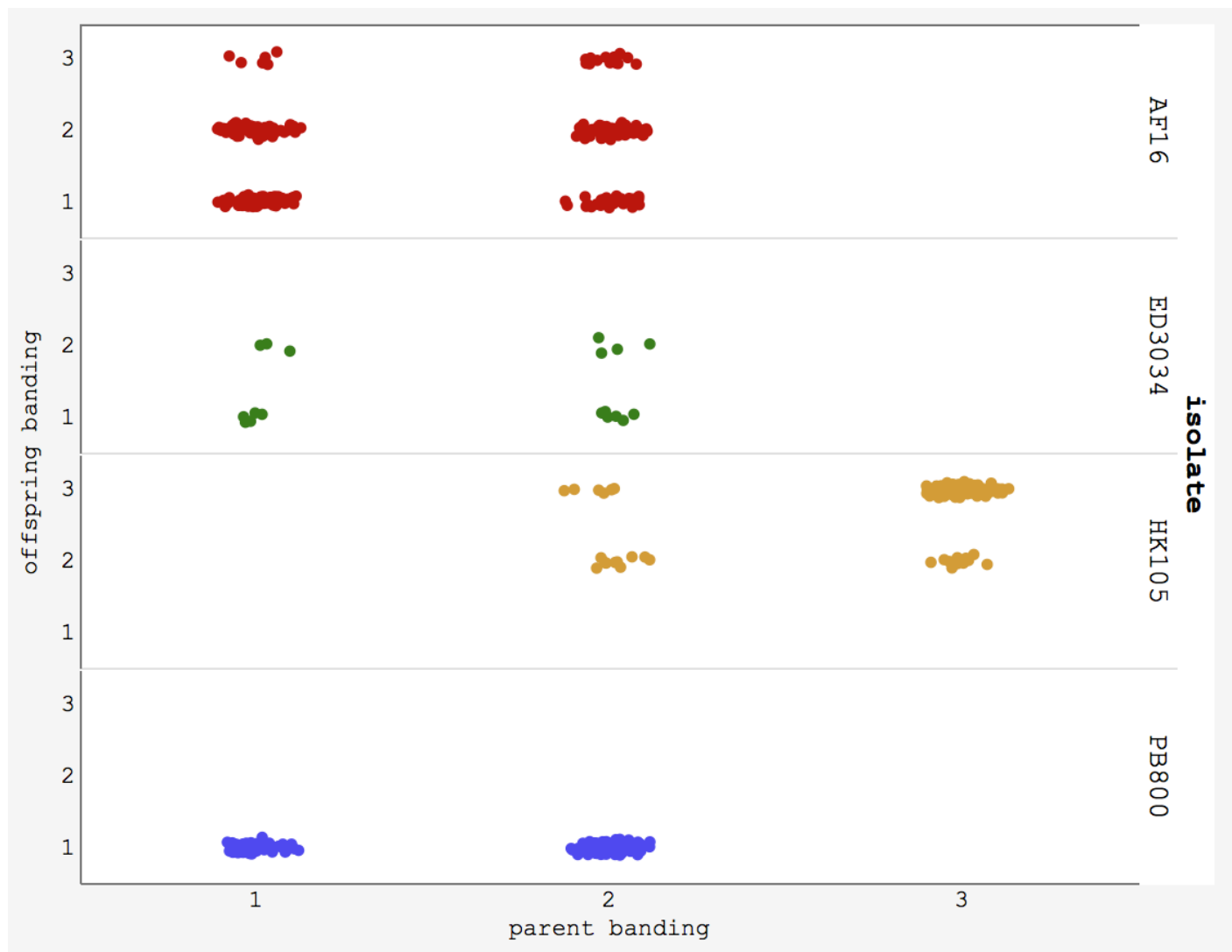

Figure 12. Parent and offspring banding correlation scatterplot by strain. Offspring nad5 $\Delta$ levels tend to be equal to or greater than those of their mothers, while PB800 exhibits lower deletion levels overall and lower rates of transmission than in other isolates. 
Table 4. qPCR mean proportion nad5s by isolate by inheritance. C+ and Cdesignations denote whether or not the isolate contains a putative compensatory mutation. 1 S.E.M. value reported. $n=$ final sample size of successful qPCR reactions per group.

\begin{tabular}{|c|c|c|c|c|c|}
\hline Isolate & Assay & Life stage & 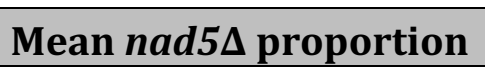 & S.E.M. & $n$ \\
\hline \multirow{2}{*}{ 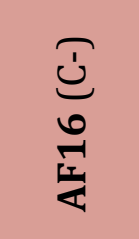 } & \multirow{2}{*}{-} & Parent & 0.2816 & 0.0544 & 10 \\
\hline & & Offspring & 0.1861 & 0.0143 & 157 \\
\hline \multirow{2}{*}{ 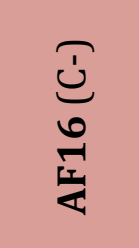 } & \multirow{2}{*}{$N$} & Parent & 0.1172 & 0.0457 & 10 \\
\hline & & Offspring & 0.0459 & 0.0045 & 160 \\
\hline \multirow{2}{*}{$\begin{array}{l}\underset{f}{\mathcal{E}} \\
8 \\
\stackrel{8}{0} \\
\stackrel{0}{0}\end{array}$} & \multirow{2}{*}{-1} & Parent & 0.3613 & 0.0399 & 10 \\
\hline & & Offspring & 0.0382 & 0.0053 & 136 \\
\hline \multirow{2}{*}{ 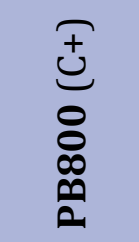 } & \multirow{2}{*}{$N$} & Parent & 0.2003 & 0.0392 & 10 \\
\hline & & Offspring & 0.0680 & 0.0056 & 160 \\
\hline
\end{tabular}

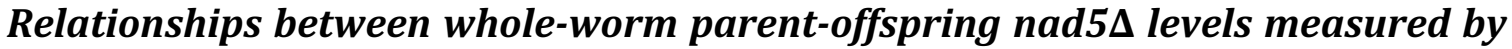

$\boldsymbol{q P C R}$. Final sample sizes for the transmission gel banding assays are provided in

Table 4. Once again, certain sample groups within certain assays (e.g., offspring 
samples in Assay 1) experienced more failed PCRS than others. Quantitative real-time PCR methods were employed on the second set of parent-L1 offspring to assess relative abundance of nad5 $\Delta$ across inheritance in whole-animal samples. Data show a marginally positively significant relationship between parent and mean offspring nad5 $\Delta$ transmission for C- AF16, and a nonsignificant relationship for C+ PB800 (Figure 13). Notably, there were two outlying AF16 families for which average offspring deletion levels were approximately $50 \%$.

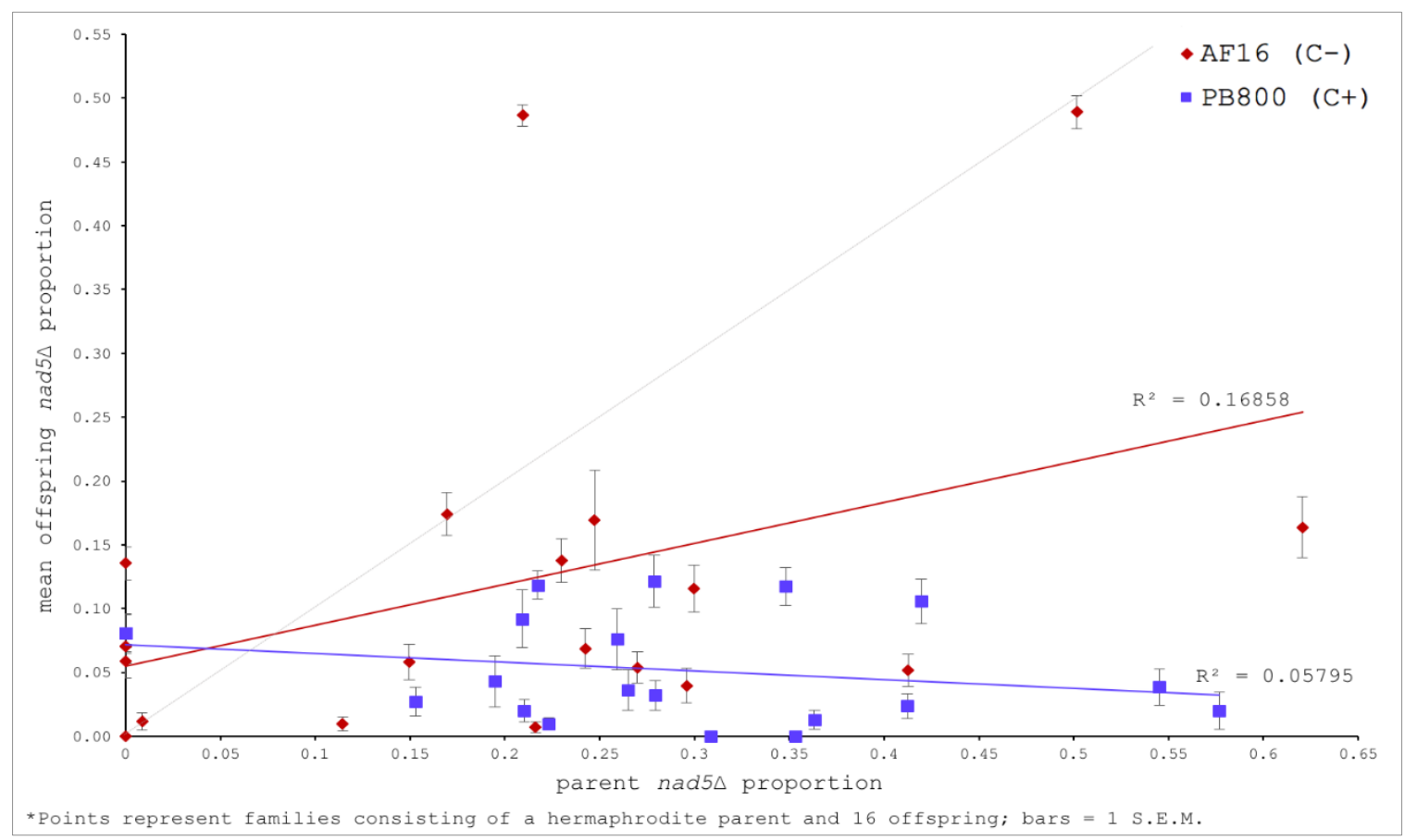

Figure 13. Bivariate plot of mean offspring nad5s by parent. qPCR data showing proportion nad5 $\Delta$ of all parent-mean offspring pairs (whole parent versus average whole offspring) for both AF16 and PB800 nematodes. Light gray projected line shows a 1:1 relationship. The red line indicates the best fit line according to standard least squares regression where $\mathrm{y}$ is mean AF16 offspring nad5 $\Delta$ level; $\mathrm{y}=0.3208 \mathrm{x}+0.0546 .\left(F_{1,18}=3.65, p\right.$ $=0.07$, non-significant intercept). The blue line indicates the same for PB800; $\mathrm{y}=-0.0686+$ 0.072. $\left(F_{1,18}=1.11, p=3.06\right.$, intercept \pm S.E.M. $\left.=0.072 \pm 0.020\right)$. 


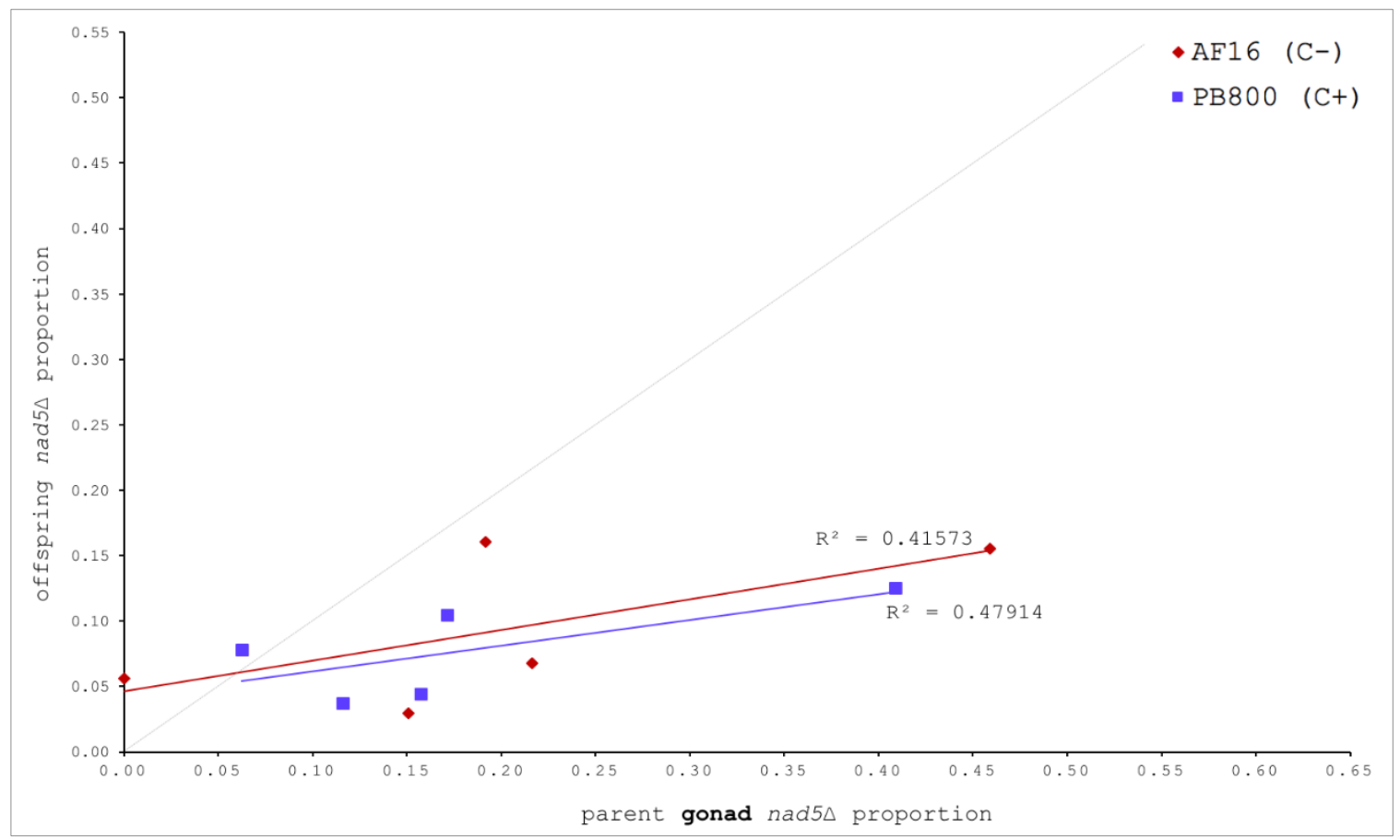

Figure 14. Bivariate plot of mean offspring nad5 $\Delta$ by parent gonad. qPCR data showing proportion nad5 $\Delta$ of all parent-mean offspring pairs (mother's gonad versus average whole offspring) for both AF16 and PB800 nematodes. Light gray projected line shows a 1:1 relationship. The red line indicates the best fit line according to standard least squares regression where $\mathrm{y}$ is mean AF16 offspring nad5 level; $\mathrm{y}=0.2347 \mathrm{x}+0.0461 .\left(F_{1,3}=2.13, p\right.$ $=2.40$, non-significant intercept). The blue line indicates the same for PB800; $\mathrm{y}=0.1968 \mathrm{x}+$ 0.0420. ( $F_{1,3}=2.76, p=1.95$, non-significant intercept $)$.

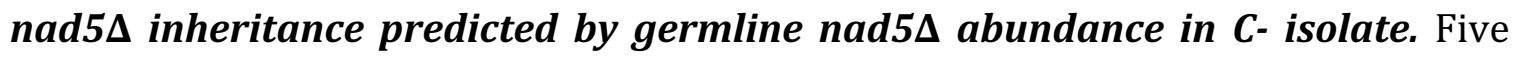
reproductive hermaphrodites of both AF16 and PB800 and 16 of each of their offspring were collected and analyzed by qPCR methods to determine relative abundance of nad5 $\Delta$ across inheritance by isolated tissue types. Parents were dissected as previously described and independent somatic and gametic samples were collected. Data show weakly positive (nonsignificant) relationships between

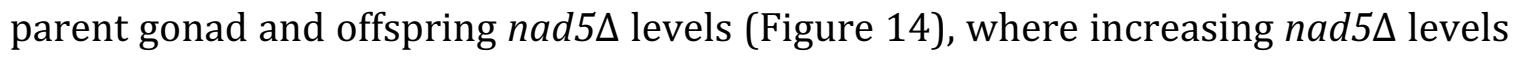
in parent gonad tissue correlates with increasing nad5s levels in whole-animal 
offspring samples for both strains. The relationship is slightly more pronounced in the AF16 isolate. Data also show weakly positive (nonsignificant) relationships

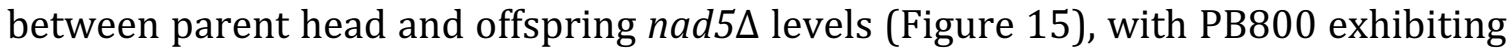
a much more truncated range in parent head \% nad5 than in AF16. Finally, Figure

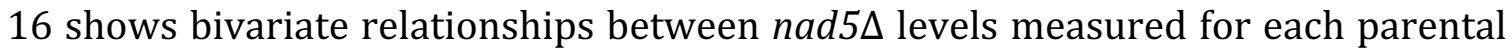
tissue type. Somatic and germline nad5 $\Delta$ levels are positively correlated within the CAF16 (Spearman's $\rho=0.900, p=0.037$ ), while no discernable relationship exists for the C+ PB800 isolate.

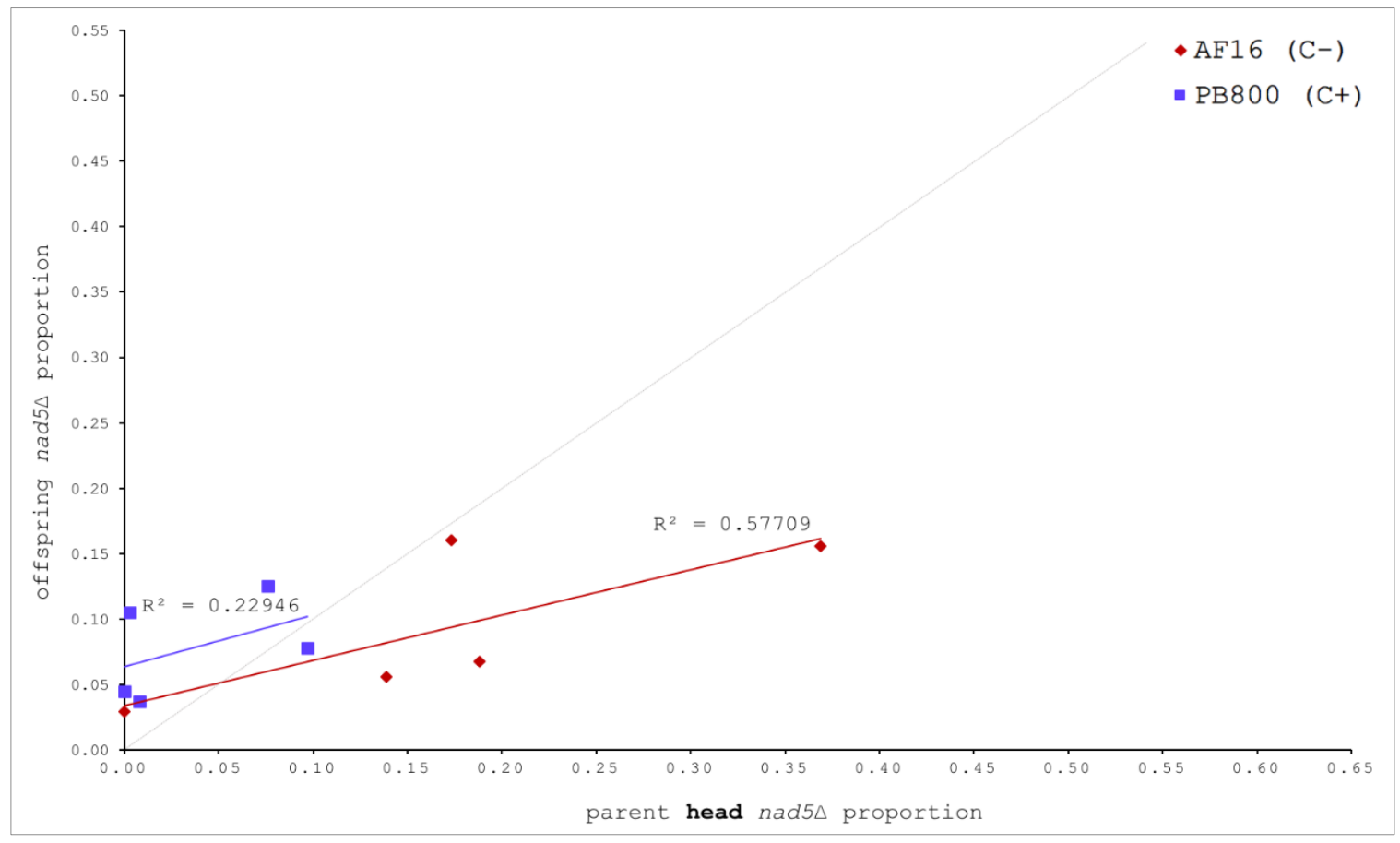

Figure 15. Bivariate plot of mean offspring nad5 $\Delta$ by parent head. qPCR data showing proportion nad5 5 of all parent-mean offspring pairs (mother's head versus average whole offspring) for both AF16 and PB800 nematodes. Light gray projected line shows a 1:1 relationship. The red line indicates the best fit line according to standard least squares regression where $\mathrm{y}$ is mean $\mathrm{AF} 16$ offspring nad5 level; $\mathrm{y}=0.347 \mathrm{x}+0.0335 .\left(F_{1,3}=4.09, p=\right.$ 0.136 , non-significant intercept). The blue line indicates the same for PB800; $y=0.3938 \mathrm{x}+$ 0.0636. $\left(F_{1,3}=0.893, p=0.414\right.$, non-significant intercept $)$. 


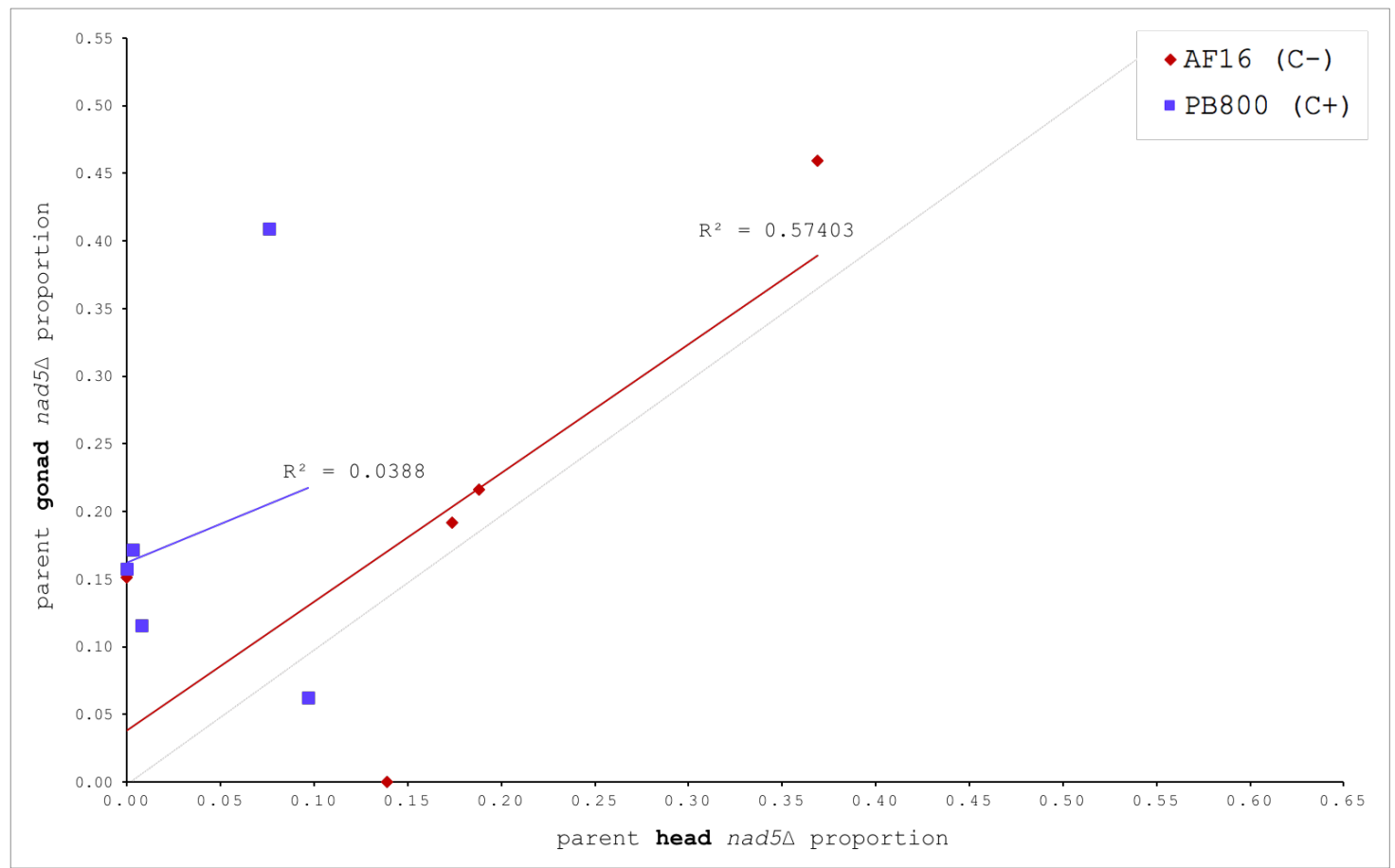

Figure 16. Bivariate plot of parent gonad nad5 5 by parent head. qPCR data showing proportion nad $5 \Delta$ for parent tissue-type pairs (mother's gonad versus head) for both AF16 and PB800 nematodes. Light gray projected line shows a 1:1 relationship. The red line indicates the best fit line according to standard least squares regression where $y$ is mean AF16 gonad nad5 level; $\mathrm{y}=0.9509 \mathrm{x}+0.0382 .\left(\mathrm{F}_{1,3}=4.04, \mathrm{p}=1.38\right.$, non-significant intercept; Spearman's $\rho=0.900, p=0.037$ ). The blue line indicates the same for PB800; $y$ $=0.5695 \mathrm{x}+0.1622 .\left(\mathrm{F}_{1,3}=0.121, \mathrm{p}=7.51\right.$, non-significant intercept $)$. 
D is c u s s i o n

Results of this thesis provide the first comprehensive assessment of naturallyoccurring mtDNA deletion dynamics during animal development and transmission. Overall, the results from both standard and quantitative PCR assays provide confirmation of the selfish nature of nad5s (Clark et al. 2012; Phillips et al. 2015) and show that compensatory mutations present in PB800 isolates may help to slow its transmission and age-related accumulation.

Age-related nad5s dynamics. Figure 11 shows that while nad5s heteroplasmy levels increased with age in all isolates tested, the increase was slowest in PB800the isolate for which initial deletion proportion was lowest. HK105, which is heavily loaded to begin with, maintained a high deletion load across development. Additionally, only AF16 experienced a major increase in nad5 $\Delta$ coincident with the L3-YA mitochondrial expansion-against our initial prediction that all isolates would exhibit such an increase. Indeed, age-related increases in deletion levels were rather gradual for the other strains with the L1 developmental stage being the least loaded

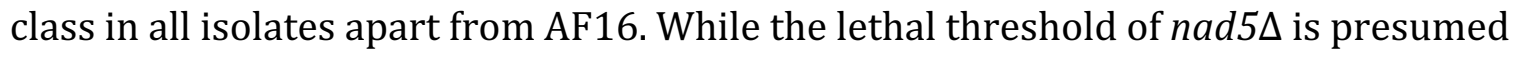
to be approximately $60 \%$ in whole adult worms, levels in L1 offspring, which should reflect maternal deletion contributions, rarely reach that level. Although L4 or YA stages tended to be the most loaded developmental classes (Table 2), the absence of a sharp increase in deletion levels at these stages is consistent with intra-individual purifying selection operating to reduce nad5 $\Delta$ frequencies. To the extent that changes 
in nad5 $\Delta$ levels observed across development were a consequence of shifts in germline rather than somatic mtDNA, they suggest that selection against nad5Lcontaining gametes may occur prior to fertilization (Bratic et al. 2009). In summary, results from the development assay suggest that the divergent sequences present within PB800 may provide some protection against deletion accumulation. Future application of qPCR technology and inclusion of more C+ and C- isolates would be revealing, perhaps exploring HK105 versus HK104 and other low-heteroplasmy Cisolates in particular.

nad5s inheritance. C+ PB800 offspring born to heteroplasmic mothers (with a deletion banding score of 2) showed a strong reversion to wildtype banding scores of 1 (Figure 12); this pattern was not observed for the C- isolates. Although C- isolate parents produced some offspring with lower deletion levels than themselves, there

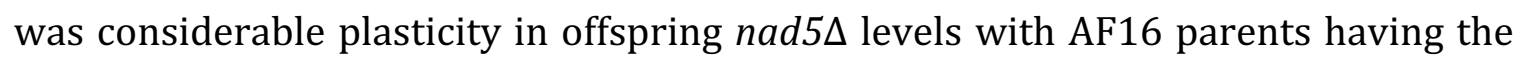
strongest tendency to produce offspring with higher deletion banding scores than themselves.

The same general result was given by the qPCR analyses; C- AF16 isolate parents showed a weak tendency to generate offspring with higher nad5 5 load than C+ PB800 isolate parents (Figure 13); however, nad54 inheritance was not well predicted by total maternal nad5s proportion in either isolate. Offspring deletion levels for both isolates tended to be much lower than maternal levels, consistent with some form of negative selection operating during transmission—a marked difference from inheritance trends of the experimental uaDf5 deletion in C. elegans, which has a 
roughly 1:1 relationship between parent and offspring deletion levels (Liau et al. 2007). This difference may suggest that different rules apply to different types of deletions and/or that uaDf5 may not be a good representation of natural mtDNA deletion dynamics as it is lab-generated and the mutant strain has had no opportunity to evolve compensatory or selective mechanisms to limit its transmission. Despite this, C- isolate AF16 exhibited a slightly more positive relationship between maternal

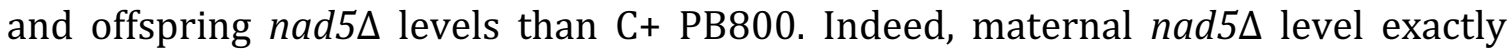
predicted mean offspring deletion levels for a few AF16 families, and two extreme

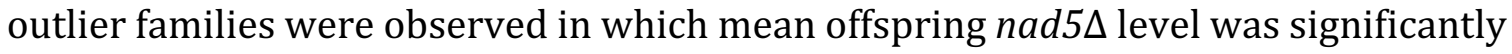
higher than the maternal level (Figure 13). These patterns are consistent with either or both: 1) more frequent nad5s transmission in AF16 (i.e., relaxed selection on nad5 $\Delta$ in the maternal AF16 germline), and 2) more frequent deletion formation during early embryogenesis in AF16 compared to PB800.

For nad5 $\Delta$ inheritance broken out by tissue type (Figures 14 and 15), mean

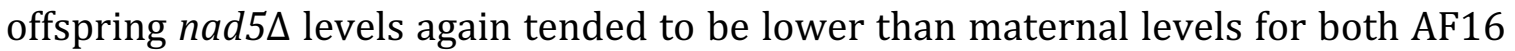
and PB800. Offspring from both isolates thus appear to be protected from rampant deletion expansion. Although mean offspring deletion levels were still poorly predicted by maternal levels measured from germline and somatic tissues (Figures 14 and 15), these maternal-offspring relationships were more positive and less variable than those revealed by the whole-worm maternal samples (Figure 13).

nad5 5 heteroplasmy in soma versus germline. $\mathrm{qPCR}$ analyses of maternal somatic and gonadal tissue nad5 levels had slightly more power to predict offspring deletion 
levels than did whole-worm maternal samples, indicating the importance of tissue dissections in future work; analyses of maternal gonad appear to be a better predictor of offspring deletion levels for both isolates. Any correlation between maternal somatic deletion level and offspring deletion level is highly unlikely to indicate an effect of somatic mitochondrial DNA inheritance, which is not known to occur in Caenorhabditid nematodes. The difference between maternal whole-worm and tissue-specific patterns likely points to variance in nad5s levels in an untested parental tissue type (e.g., gut) present within the whole-worm maternal samples. Interestingly, the data range for somatic (head) nad5 level (Figure 15) in PB800 is contracted compared to gonad (Figure 14). This may be a consequence of our small sample size failing to capture the full range of PB800 nad5 levels, but an alternative interpretation is that less frequent deletion formation (and possibly more efficient purifying selection) occurred in the mitochondria-rich pharynx of PB800 compared to AF16, and suggests that any protective function of compensatory mutation against nad5 $\Delta$ is more important to high energy-demand somatic tissues than to germline tissues. Selecting a less energetically active somatic tissue type for analysis may have yielded different results.

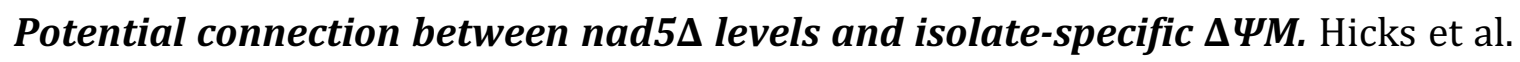
(2012) found that mitochondrial inner membrane potential $(\Delta \Psi \mathrm{M})$ predicted $C$. briggsae clade membership (clades I \& II) such that clade I (previously Tropical) isolates exhibited lower $\Delta \Psi M$. They hypothesized that these isolates, which are adapted to warmer temperatures (Prasad et al. 2011), may adaptively maintain lower 
membrane potential for the purpose of limiting mitochondrial ROS production (Brand 2000). Brand posited that uncoupling OXPHOS mechanisms, which would reduce $\Delta \Psi \mathrm{M}$, could provide a mechanism for reducing ROS production in addition to thermogenesis. If true, however, the lower $\triangle \Psi M$ associated with such uncoupling would also negatively affect mitochondrial fission-fusion dynamics and mitophagy cycles (Twig et al. 2008), which could reduce the ability of cells to clear damaged mtDNA. Applied to this project, clade I isolates could be less efficient at clearing deletion-bearing mtDNA; this could help to account for the higher nad5A heteroplasmy load and variability (apart from the absence of the compensatory mutations) in AF16 and ED3034 compared to clade II isolates. 


\section{H A P T E R : Co n c l u s i o n}

This thesis aimed to provide deeper insight into the patterns of mtDNA deletion transmission and age-associated dynamics, and discover clues regarding the relative importance of different evolutionary forces-mutation and selection-in shaping these dynamics. It was the first project of its type to survey mutation dynamics and heteroplasmy levels of a large-scale mtDNA deletion that segregates within natural populations. Thus, my work could serve to further develop Caenorhabditis briggsae for use as an experimental model of human mtDNA deletion dynamics-a muchneeded supplement to human pedigree and cell culture studies (Russell et al. 2018).

All previous work performed on nad5s (Howe and Denver 2008; Howe et al. 2010; Clark et al. 2012; Phillips et al. 2015) explored the effects of varying genetic drift conditions on nad5 $\Delta$ across multiple generations. Each of these studies showed that, under conditions of extreme genetic drift, nad5 $\Delta$ deletion frequency increased from initial isolate-specific levels up to a threshold level. This increase was especially pronounced for isolates lacking compensatory mutations. Furthermore, Phillips et al. (2015; Fig. 5) demonstrated that reducing the effects of drift (and increasing the effects of selection) by increasing experimental population size limited nad5 $\Delta$ accumulation across generations. Results of these prior studies are consistent with: 1) nad5s having a replication advantage, 2) purifying (truncation) selection operating to maintain nad5 $\Delta$ levels below a lethal threshold, and 3) compensatory mutations reducing the likelihood of new nad5 $\Delta$ formation in $\mathrm{C}+$ isolates; however, 
they could not distinguish the relative impact of these mechanisms in determining

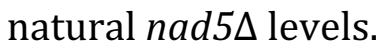

My work added to the above body of knowledge by studying nad5 $\Delta$ levels across a single generation. My results differed from those above in that nad5 $\Delta$ levels did not show a strong tendency toward increase; rather, hermaphrodite mothers tended to produce offspring with fewer deletion-bearing mtDNA genomes than themselves across the single generation assayed. This was true for both C+ and Cisolates, and suggests that any replication advantage nad5s may have during maternal germline development is opposed by another force during mtDNA transmission to offspring. Konrad et al. (2017) estimated the C. elegans mitochondrial bottleneck size at $62 \mathrm{mtDNA}$ copies transmitted per C. elegans hermaphrodite. If the same is approximately true for $C$. briggsae, this number is likely sufficient for effective selection against deletion-bearing genomes. The nature of the selective force remains unclear, but could be related to a mechanism involved during the normal period of physiological apoptosis occurring in the hermaphrodite gonad (Raiders et al. 2018). This natural process is thought to provide cytoplasmic material for developing oocytes while eliminating aberrant cells; i.e., multinucleate germ cells. As suggested by Raiders et al. 2018, it is feasible that this process also serves to selectively eliminate damaged mitochondria on the basis of morphology, which is known to be related to mitochondrial health; e.g., membrane potential (Ishihara et al. 2003; Okamoto and Shaw 2005; Hicks et al. 2012).

Also consistent with previous studies (Howe and Denver 2008; Estes et al. 2011; Phillips et al. 2015), my data indicate a potentially large role for compensatory 
mutations $(\mathrm{C}+)$ in limiting and/or reducing nad5s levels—separate from any effects of mitochondrial quality control via mitophagy (Twig et al. 2008; Mishra and Chan 2014). Taken together, these results suggest that population-level nad5 $\Delta$ frequencies are likely defined by a balance between recurrent deletion formation as well as selection occurring at gametic and individual levels. Specifically, nad5 5 , promoted by the flanking repeat sequences, forms at some rate that is limited by the presence of the compensatory mutations in $\mathrm{C}+$ isolates; and, in both types of isolates, truncation selection limits nad5 accumulation above a certain lethal threshold level. This threshold level as well as the exact selective force likely differs between germline and soma.

A major caveat to the findings of this project is that the results are correlative, and additional nuclear and mitochondrial genetic variation beyond nad5 5 present within C. briggsae isolates is unaccounted for; thus, results may be confounded by potential effects of background genetic variation and phylogentics. Additional limitations may result from exclusively using primers specific to the canonical nad5 $\Delta$ deletion; we may be missing other deletions affecting the same region (Phillips et al. 2015). However, such deletions were observed previously only in C. briggsae populations evolved for multiple generations under strong drift conditions (Phillips et al. 2015; Fig. 5). Finally, in agreement with previous finding from our group, this

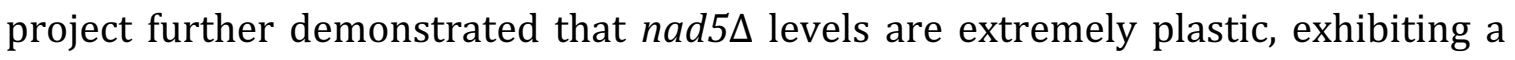
high degree of variance among individuals and isolates. Thus, other caveats to these conclusions include considerable inter-individual and intra-family variability in nad5 levels, particularly among maternal individuals within isolates (Tables 3 and 
4). Assaying a larger set of samples including more C+ and C- individuals might reveal a stronger signal.

Future research. Continued work on this project will focus on increasing sample sizes of all qPCR assays (including whole worm and parent dissections) to increase statistical power. Completion of standard PCR assays using C+ EG4181 will provide more insight into the role of compensatory mutations in protecting from nad5 $\Delta$ formation and transmission. Additionally, future dissections of maternal hermaphrodites will involve segregating tissues from head, gonad, and gut to assess the major source of mtDNA variance within the individuals.

Producing inbred lines of $C$. briggsae natural isolates with high or low heteroplasmy levels (Dolgin et al. 2007) would provide further insight into effects of

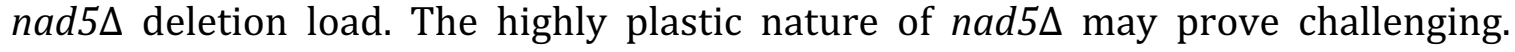
Cytoplasmic-nuclear hybrid (cybrid) strains generated with varying nad5s heteroplasmy levels on uniform genetic backgrounds (Estes et al. 2011) could also assist in disentangling the effects of nad5 $\Delta$ variation among isolates from the effects of among-isolate nuclear variation, but mitochondrial paternal leakage observed to occur in certain C. briggsae isolates poses a potential limitation (Ross et al. 2016). Germline apoptosis assays using acridine orange staining (Lant and Derry 2013) in

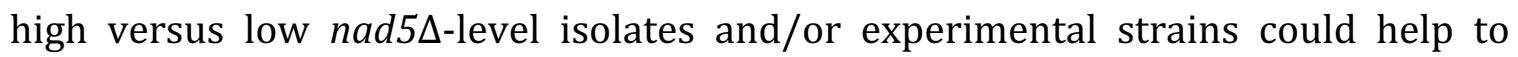
identify specific selective mechanisms that may be occurring between generations. Finally, future pharmacological manipulations could be used to explore the effects of 
mitochondrial fission-fusion-mitophagy or other processes on preventing mitochondrial deletion expansion. 
R E F E R E N C E S

Albertini AM, Hofer M, Calos MP, Miller JH. 1982. On the formation of spontaneous deletions: The importance of short sequence homologies in the generation of large deletions. Cell 29:319-328.

Baer CF, Shaw F, Steding C, Baumgartner M, Hawkins A, Houppert A, Mason N, Reed M, Simonelic K, Woodard W, et al. 2005. Comparative evolutionary genetics of spontaneous mutations affecting fitness in rhabditid nematodes. Proc. Natl. Acad. Sci. U. S. A. 102:5785-5790.

Brand M. 2000. Uncoupling to survive? The role of mitochondrial inefficiency in ageing. Exp. Gerontol. 35:811-820.

Bratic I, Hench J, Henriksson J, Antebi A, Bürglin TR, Trifunovic A. 2009. Mitochondrial DNA level, but not active replicase, is essential for Caenorhabditis elegans development. Nucleic Acids Res. 37:1817-1828.

Brown D, Herbert M, Lamb V, Chinnery P, Taylor R, Lightowlers R, Craven L, Cree L, Gardner J, Turnbull D. 2006. Transmission of mitochondrial DNA disorders: possibilities for the future. Lancet 368:87-89.

Chan CCW, Liu VWS, Lau EYL, Yeung WSB, Ng EHY, Ho PC. 2006. Mitochondrial DNA content and 4977 bp deletion in unfertilized oocytes. Mol. Hum. Reprod. 11:843-846.

Chen T, He J, Shen L, Fang H, Nie H, Jin T, Wei X, Xin Y, Jiang Y, Li H, et al. 2011. The mitochondrial DNA 4,977-bp deletion and its implication in copy number alteration in colorectal cancer. BMC Med. Genet. 12:8.

Chen XJ. 2013. Mechanism of Homologous Recombination and Implications for Aging-Related Deletions in Mitochondrial DNA. Microbiol. Mol. Biol. Rev. 77:476-496.

Chinnery PF, Johnson MA, Wardell TM, Singh-Kler R, Hayes C, Brown DT, Taylor RW, Bindoff LA, Turnbull DM. 2000. The epidemiology of pathogenic mitochondrial DNA mutations. Ann. Neurol. 48:188-193.

Chinnery PF, Thorburn DR, Samuels DC, White SL, Dahl H-HM, Turnbull DM, Lightowlers RN, Howell N. 2000. The inheritance of mitochondrial DNA heteroplasmy: random drift, selection or both? Trends Genet. 16:500-505.

Clark KA, Howe DK, Gafner K, Kusuma D, Ping S, Estes S, Denver DR. 2012. Selfish 
little circles: Transmission bias and evolution of large deletion-bearing mitochondrial DNA in caenorhabditis briggsae nematodes. PLoS One 7:1-8.

Dani SU, Dani MAC, Simpson AJG. 2003. The common mitochondrial DNA deletion mtDNA4977: Sheding new light to the concept of a tumor suppressor mutation. Med. Hypotheses 61:60-63.

Dolgin ES, Charlesworth B, Baird SE, Cutter AD. 2007. Inbreeding and outbreeding depression in Caenorhabditis nematodes. Evolution. 61:1339-1352.

Estes S, Coleman-Hulbert AL, Hicks K a, de Haan G, Martha SR, Knapp JB, Smith SW, Stein KC, Denver DR. 2011. Natural variation in life history and aging phenotypes is associated with mitochondrial DNA deletion frequency in Caenorhabditis briggsae. BMC Evol. Biol. 11:11.

Felsenstein J. 1974. The evolutionary advantage of recombination. Genetics 78:737756.

Gabriel W, Lynch M, Burger R. 1993. Muller's Ratchet and Mutational Meltdowns. Evolution. 47:1744-1757.

Gomez-Gomez ME. 2017. Mitochondria DNA Mutations and Mitochondrial Diseases. In: Zapico SC, editor. Mechanisms Linking Aging, Diseases and Biological Age Estimation. p. 201-214.

Hauswirth WW, Laipis PJ. 1982. Mitochondrial DNA polymorphism in a maternal lineage of Holstein cows. Proc. Natl. Acad. Sci. U. S. A. 79:4686-4690.

Hicks KA, Howe DK, Leung A, Denver DR, Estes S. 2012. In Vivo Quantification Reveals Extensive Natural Variation in Mitochondrial Form and Function in Caenorhabditis briggsae. PLoS One 7.

Howe DK, Baer CF, Denver DR. 2010. High rate of large deletions in Caenorhabditis briggsae mitochondrial genome mutation processes. Genome Biol. Evol. 2:29-38.

Howe DK, Denver DR. 2008. Muller's Ratchet and compensatory mutation in Caenorhabditis briggsae mitochondrial genome evolution. BMC Evol. Biol. 8:62.

Hsieh RH, Tsai NM, Au HK, Chang SJ, Wei YH, Tzeng CR. 2002. Multiple rearrangements of mitochondrial DNA in unfertilized human oocytes. Fertil. Steril. 77:1012-1017.

Hudson G, Chinnery PF. 2006. Mitochondrial DNA polymerase- $\gamma$ and human disease. 
Hum. Mol. Genet. 15:244-252.

Ishihara N, Jofuku A, Eura Y, Mihara K. 2003. Regulation of mitochondrial morphology by membrane potential, and DRP1-dependent division and FZ01-dependent fusion reaction in mammalian cells. Biochem. Biophys. Res. Commun. 301:891-898.

Kaukonen J, Juselius JK, Tiranti V, Kyttala A, Zeviani M, Comi GP, Keranen S, Peltonen L, Suomalainen A. 2000. Role of adenine nucleotide translocator 1 in mtDNA maintenance. Science. 289:782-785.

Konrad A, Thompson O, Waterston RH, Moerman DG, Keightley PD, Bergthorsson U, Katju V. 2017. Mitochondrial mutation rate, spectrum and heteroplasmy in Caenorhabditis elegans spontaneous mutation accumulation lines of differing population size. Mol. Biol. Evol. 34:1319-1334.

Krishnan KJ, Reeve AK, Samuels DC, Chinnery PF, Blackwood JK, Taylor RW, Wanrooij S, Spelbrink JN, Lightowlers RN, Turnbull DM. 2008. What causes mitochondrial DNA deletions in human cells? Nat Genet 40:275-279.

Lant B, Derry WB. 2013. Methods for detection and analysis of apoptosis signaling in the C. elegans germline. Methods 61:174-182.

Lemire B. 2005. Mitochondrial genetics. WormBook:1-10.

Lewis SC, Joers P, Willcox S, Griffith JD, Jacobs HT, Hyman BC. 2015. A Rolling Circle Replication Mechanism Produces Multimeric Lariats of Mitochondrial DNA in Caenorhabditis elegans. PLoS Genet. 11:1-16.

Liau WS, Gonzalez-Serricchio AS, Deshommes C, Chin K, LaMunyon CW. 2007. A persistent mitochondrial deletion reduces fitness and sperm performance in heteroplasmic populations of C. elegans. BMC Genet. 8:1-11.

Lynch M, Conery J, Burger R. 1995. Mutation Accumulation and the Extinction of Small Populations. Am. Nat. 146:489-518.

Lynch M, Conery J, Burger R. 2012. Mutational Meltdowns in Sexual Populations. Society for the Study of Evolution. 49:1067-1080.

Ma H, O'Farrell PH. 2016. Selfish drive can trump function when animal mitochondrial genomes compete. Nat. Genet. 48:798-802.

Mishra P, Chan DC. 2014. Mitochondrial dynamics and inheritance during cell division, development and disease. Nat. Rev. Mol. Cell Biol. 15:634-646. 
Mita S, Rizzuto R, Moraes CT, Shanske S, Arnaudo E, Fabrizi GM, Koga Y, Dimauro S, Schon EA. 1990. Recombination via flanking direct repeats is a major cause of large-scale deletions of human mitochondrial DNA. Nucleic Acids Res. 18:561-567.

Moraes CT, Kenyon L, Hao H. 1999. Mechanisms of human mitochondrial DNA maintenance: the determining role of primary sequence and length over function. Mol. Biol. Cell 10:3345-3356.

Muller HJ. 1964. The Relation of Recombination to Mutational Advance. Mutat. Res. 1:2-9.

Nie H, Shu H, Vartak R, Milstein AC, Mo Y, Hu X, Fang H, Shen L, Ding Z, Lu J, et al. 2013. Mitochondrial Common Deletion, a Potential Biomarker for Cancer Occurrence, Is Selected against in Cancer Background: A Meta-Analysis of 38 Studies. PLoS One 8:15-19.

Okamoto K, Shaw JM. 2005. Mitochondrial Morphology and Dynamics in Yeast and Multicellular Eukaryotes. Annu. Rev. Genet. 39:503-536.

De Paula WBM, Agip ANA, Missirlis F, Ashworth R, Vizcay-Barrena G, Lucas CH, Allen JF. 2013. Female and male gamete mitochondria are distinct and complementary in transcription, structure, and genome function. Genome Biol. Evol. 5:1969-1977.

Phillips AF, Millet AR, Tigano M, Dubois SM, Crimmins H, Babin L, Charpentier M, Piganeau M, Brunet E, Sfeir A. 2017. Single-Molecule Analysis of mtDNA Replication Uncovers the Basis of the Common Deletion. Mol. Cell 65:527538.

Phillips WS, Coleman-Hulbert AL, Weiss ES, Howe DK, Ping S, Wernick RI, Estes S, Denver DR. 2015. Selfish Mitochondrial DNA Proliferates and Diversifies in Small, but not Large, Experimental Populations of Caenorhabditis briggsae. Genome Biol. Evol. 7:2023-2037.

Pitceathly RDS, Rahman S, Hanna MG. 2012. Single deletions in mitochondrial DNA Molecular mechanisms and disease phenotypes in clinical practice. Neuromuscul. Disord. 22:577-586.

Pitkanen S, Robinson BH, Pitkänen S, Robinson BH. 1996. Mitochondrial complex I deficiency leads to increased production of superoxide radicals and induction of superoxide dismutase. J. Clin. Invest. 98:345-351.

Poulton J, Deadman ME, Ramacharan S, Gardiner RM. 1991. Germ-line deletions of mtDNA in mitochondrial myopathy. Am. J. Hum. Genet. 48:649-653. 
Prasad A, Croydon-Sugarman MJF, Murray RL, Cutter AD. 2011. Temperaturedependent fecundity associates with latitude in caenorhabditis briggsae. Evolution. 65:52-63.

Raiders SA, Eastwood MD, Bacher M, Priess JR. 2018. Binucleate germ cells in Caenorhabditis elegans are removed by physiological apoptosis. PLoS Genet. 14:1-47.

Reeve AK, Krishnan KJ, Turnbull D. 2008. Mitochondrial DNA mutations in disease, aging, and neurodegeneration. Ann. N. Y. Acad. Sci. 1147:21-29.

Ross JA, Howe DK, Coleman-Hulbert A, Denver DR, Estes S. 2016. Paternal mitochondrial transmission in intra-species Caenorhabditis briggsae hybrids. Mol. Biol. Evol. 33:3158-3160.

Russell OM, Fruh I, Rai PK, Marcellin D, Doll T, Reeve A, Germain M, Bastien J, Rygiel KA, Cerino R, et al. 2018. Preferential amplification of a human mitochondrial DNA deletion in vitro and in vivo. Sci. Rep. 8:1-10.

Samuels DC. 2004. Mitochondrial DNA repeats constrain the life span of mammals. Trends Genet. 20:226-229.

Sato A, Nakada K, Shitara H, Kasahara A, Yonekawa H, Hayashi JI. 2007. Deletionmutant mtDNA increases in somatic tissues but decreases in female germ cells with age. Genetics. 177:2031-2037.

Sato M, Sato K. 2011. Degradation of paternal mitochondria by fertilizationtriggered autophagy in C. elegans embryos. Science. 334:1141-1144.

Shitara H, Kaneda H, Sato A, Inoue K, Ogura A, Yonekawa H, Hayashi JI. 2000. Selective and continuous elimination of mitochondria microinjected into mouse eggs from spermatids, but not from liver cells, occurs throughout emborogenesis. Genetics 156:1277-1284.

Shoffner JM, Lott MT, Voljavec AS, Soueidan SA, Costigan DA, Wallace DC. 1989. Spontaneous Kearns-Sayre/chronic external ophthalmoplegia plus syndrome associated with a mitochondrial DNA deletion: a slip-replication model and metabolic therapy. Proc. Natl. Acad. Sci. 86:7952-7956.

Spelbrink JN, Li FY, Tiranti V, Nikali K, Yuan QP, Tariq M, Wanrooij S, Garrido N, Comi G, Morandi L, et al. 2001. Human mitochondrial DNA deletions associated with mutations in the gene encoding Twinkle, a phage T7 gene 4like protein localized in mitochondria. Nat. Genet. 28:223-231. 
Stewart JB, Chinnery PF. 2015. The dynamics of mitochondrial DNA heteroplasmy: implications for human health and disease. Nat. Rev. Genet. 16:530-42.

Stewart JB, Larsson N-G. 2014. Keeping mtDNA in Shape between Generations. PLoS Genet. 10:1-7.

Tsang WY, Lemire BD. 2002. Stable heteroplasmy but differential inheritance of a large mitochondrial DNA deletion in nematodes. Biochem. Cell Biol. 80:645654.

Twig G, Hyde B, Shirihai OS. 2008. Mitochondrial fusion, fission and autophagy as a quality control axis: The bioenergetic view. Biochim. Biophys. Acta Bioenerg. 1777:1092-1097.

Vielhaber S, Kunz D, Winkler K, Wiedemann FR, Kirches E, Feistner H, Heinze HJ, Elger CE, Schubert W, Kunz WS. 2000. Mitochondrial DNA abnormalities in skeletal muscle of patients with sporadic amyotrophic lateral sclerosis. Brain 123:1339-48.

Wallace DC. 2016. Genetics: Mitochondrial DNA in evolution and disease. Nature 535:498-500.

Wallace DC, Chalkia D. 2013. Mitochondrial DNA genetics and the heteroplasmy conundrum in evolution and disease. Cold Spring Harb. Perspect. Med. 3:147. 